\title{
Literatur in vereinfachter Sprache: Einfachheit und literarische Ästhetik
}

\author{
Mathilde Hennig • Joachim Jacob
}

Angenommen: 2. Februar 2022 / Online publiziert: 24. Februar 2022

(C) Der/die Autor(en) 2022

Zusammenfassung Der naheliegenden Annahme, dass die Rahmenbedingungen Leichter und Einfacher Sprache erhebliche Grenzen für eine literarästhetische Gestaltung von Texten setzen, steht eine inzwischen stark verbreitete Praxis der Produktion literarischer Texte in Leichter und Einfacher Sprache (und zwar sowohl als Übersetzungen vorliegender literarischer Werke als auch als Eigenproduktionen) gegenüber. Der Artikel nimmt das zum Anlass, die Frage, inwieweit Einfachheit und Literarästhetik aufeinander beziehbar sind, aus einer linguistisch-literaturwissenschaftlich interdisziplinären Perspektive zu diskutieren. Der Artikel stellt Überlegungen zu Einfachheit und Komplexität aus literaturwissenschaftlicher und linguistischer Perspektive gegenüber und setzt sie in Beziehung zu den vorliegenden Regelwerken zu Leichter Sprache sowie Empfehlungen zum Verfassen von Literatur in Einfacher Sprache. Ausgehend von einem einfachen Grundverständnis einer Komplexität-Simplizität-Skala, das von einem Mehr vs. Weniger von Komplexitätsmerkmalen ausgeht, diskutiert der Beitrag, welche literarästhetischen Merkmale für Analysen dieser Art in Frage kommen. Ein exemplarischer Blick auf den kreativen Umgang mit Einfachheit in drei Textbeispielen rundet die Überlegungen ab.

Schlüsselwörter Leichte Sprache $\cdot$ Literarästhetik · Komplexität · Einfache Sprache

Mathilde Hennig $(\bowtie) \cdot$ Joachim Jacob

Fachbereich 05, Institut für Germanistik, Justus-Liebig-Universität Gießen, Gießen, Deutschland E-Mail: Mathilde.hennig@germanistik.uni-giessen.de

Joachim Jacob

E-Mail: Joachim.Jacob@germanistik.uni-giessen.de 


\title{
Literature in Easy-to-Read Language: Simplicity and Literary Aesthetics
}

\begin{abstract}
Whereas on the one hand it seems plausible to assume that ETR Languages provide unfavourable conditions for the production of aesthetic texts, literature in ETR German (translations of existing literary works as well as newly created texts) becomes more and more common at the moment. Proceeding from this, the article discusses the question of how simplicity and aesthetics may interact from an interdisciplinary linguistic and literary point of view. The article contrasts linguistics and literary aspects of simplicity and complexity and relates them to the existing guidelines for ETR-texts as well as recommendations for producing literary texts in ETR German. Based on a rather simple understanding of a scale of simplicity and complexity that just models the >more < or >less $<$ of complexity features, the article deals with the question which aesthetic features are appropriate for analyses like this. The reflections are being completed by exemplarily discussing the creative use of simplicity in three examples of literary texts.
\end{abstract}

Keywords Easy-to-Read Language $\cdot$ Literature and Aesthetics · Complexity

\section{Einleitung}

Als Maximalfall vereinfachter Sprache begegnet uns die sogenannte $>$ Leichte Sprache ${ }^{1}$ als Kind unserer Zeit: Wenn in einer Gesellschaft erhöhter Wert darauf gelegt wird, dass niemand aufgrund individueller gesundheitlicher, kognitiver oder sonstiger Voraussetzungen benachteiligt wird, ist es folgerichtig, die Teilhabe an den einschlägigen Praktiken einer literalisierten Gesellschaft zum Gegenstand von Teilhabebemühungen $\mathrm{zu}$ machen. Und $\mathrm{zu}$ diesen gehören auch literarische Texte: »Alle haben ein Recht auf Kunst und Literatur«, heißt es im Nachwort zu einer im Frühjahr 2020 erschienenen Anthologie von »Literatur in Einfacher Sprache« (Hückstädt 2020, S. 258). Leichte Sprache reagiert einerseits auf im Kontext der Teilhabebestrebungen entstehende praktische Bedürfnisse, andererseits auf gesetzliche Vorhaben zur Barrierefreiheit (Bredel/Maaß 2016, S. $68 \mathrm{ff}$.). Als bewusster Eingriff in die Sprache ist Leichte Sprache unweigerlich Gegenstand der Sprachkritik (vgl. Bredel/Maaß 2016, S. 45 ff.; Bock 2015). Andererseits ist Leichte Sprache eine empirische Tatsache. Dass das Konzept der Leichten Sprache dennoch Anlass für vielfältige Diskussionen bietet, liegt einerseits vermutlich an seinem stark normativen Impetus, andererseits daran, dass seine Realisierung bei seinen

\footnotetext{
1 >Leichte Sprache< und >Einfache Spracheく sind folgendermaßen zu unterscheiden (Bredel/Maaß 2016, S. 526 ff.): >Leichte Sprache< bildet quasi den Maximalpol der Bemühungen um sprachliche Einfachheit; >Einfache Sprache< ist eine flexiblere, weniger stark reglementierte Variante. Da beide Erscheinungsformen vereinfachter Sprache sind, sind die Übergänge recht fließend. Das gilt natürlich auch bzw. gerade für literarische Texte in vereinfachter Sprache. Wir berücksichtigen im Folgenden die Unterscheidung von >Leichter < und >Einfacher < Sprache, soweit die Autoren explizit auf sie rekurrieren, und sprechen ansonsten von > vereinfachter $<$ Sprache, wenn wir beide Formen meinen.
} 
Kritikern insbesondere »als ästhetisch inadäquat und das Sprachgefühl verletzend wahrgenommen« wird (Bredel/Maaß 2016, S. 54).

Konstitutiv für Leichte Sprache sind Regelwerke (etwa das Regelwerk des »Netzwerk Leichte Sprache« oder auch die Barrierefrei-Informationstechnik-Verordnung bzw. BITV; zu einem ausführlichen und kritischen Überblick siehe Bredel/Maaß 2016, S. 82 ff.), die in ihrer Normativität (etwa »Benutzen Sie immer die gleichen Wörter für die gleichen Dinge«, »Vermeiden Sie den Genitiv«, »Vermeiden Sie den Konjunktiv«, zitiert nach Bredel/Maaß 2016, S. 520-523) keinerlei Spielraum lassen. Sie sind deshalb zu Recht Gegenstand der Kritik, da gerade die hohe Heterogenität der Zielgruppen (vgl. Bredel/Maaß 2016, S. 139 ff.; Baumert 2016, S. 101 ff.) eigentlich eine flexible Handhabung erfordert. Außerdem wurde immer wieder das Fehlen empirischer Studien beklagt (Bock/Lange/Fix 2017, S. 19; Christmann 2017, S. 47), die dringend notwendig sind, um beurteilen zu können, inwiefern die Leichte-Sprache-Angebote tatsächlich die Adressaten erreichen und somit die Teilhabe an der literalisierten Welt verbessern.

Inzwischen liegen insbesondere dank des Leipziger Projekts »Leichte Sprache im Arbeitsleben « erste empirische Studien vor (bspw. Bock 2017, 2018; Bock/Lange 2017; Lange 2019), die u.a. einige der in den genannten Regelwerken fixierten grammatischen Regeln zum Formulieren von Texten in Leichter Sprache auf den Prüfstand stellen. Der im Leipziger Kontext entstandene Sammelband »>Leichte Sprache< im Spiegel theoretischer und angewandter Forschung « (Bock/Fix/Lange 2017) bietet eine anregende Grundlage für die weiterführende linguistische Forschung, die die Bandbreite an Zugriffsperspektiven auf den Gegenstand widerspiegelt: Von Sprachkritik über Diskurs- und Politolinguistik sowie Verstehensforschung und Textlinguistik bis hin zur Sprachdidaktik. Dabei ist allerdings mit Christmann (2017) ausdrücklich darauf hinzuweisen, dass Textverstehen und Textverständlichkeit ohnehin bereits über eine etablierte Forschungstradition verfügen (zu einem Überblick vgl. Christmann/Groeben (1996)).

Einen zentralen Beitrag zur linguistischen und übersetzungswissenschaftlichen Aufarbeitung des Konzepts leistet der im Dudenverlag erschienene Band Leichte Sprache. Theoretische Grundlagen. Orientierung für die Praxis von Ursula Bredel und Christiane Maaß (2016). Das Buch »leistet wichtige Grundlagenarbeitet « und »zeigt Möglichkeiten der Anwendung des einschlägigen interdisziplinären Wissens auf den Gegenstand, macht auf Forschungsdesiderate aufmerksam und weist auf weitere Forschungsfelder hin.«(Bock/Fix 2017, S. 132) In diesem Sinne wird es sicherlich für längere Zeit den Status eines Grundlagenwerks für sich beanspruchen können. Als Leitprinzipien Leichter Sprache benennen Bredel/Maaß »Proximität« als »Orientierung am unmittelbaren Ich-Hier-Jetzt des Lesers« (2016, S. 517), »maximale Explizitheit« sowie »Kontinuität«: konstante Verwendung prototypischer Ausdrucksmittel, strukturelle Wiederholungsgenauigkeit und chronologische Themenentwicklung (2016, S. 518).

Im Kontext der Diskussion des Prinzips der Kontinuität äußern sich Bredel/Maaß wie folgt auch zur Frage, inwieweit Leichte Sprache zugänglich für literarische Texte ist: 
Mit dem Prinzip der Kontinuität wird die ästhetische Dimension von Texten, die in der Standardsprache etwa durch das bewusste Herbeiführen von Mehrdeutigkeit, durch Variantenreichtum, durch das Aufbrechen von Logik und Chronologie, aber auch durch selbstbezügliche Sprachspiele ausgezeichnet ist, gekappt. Versteht man Ästhetik insgesamt als ein Experimentieren mit Sprache, ist die Realisierung der poetischen Sprachfunktion (Jakobson) mit Leichter Sprache konstitutiv unerreichbar [...]. Folgt man den hier vorgetragenen Überlegungen [von Inclusion Europe, M.H. und J.J.], sind der Übersetzung, aber auch der Produktion von literarästhetischen Texten in Leichter Sprache enge Grenzen gesetzt. (Bredel/Maaß 2016, S. $518 \mathrm{f}$.)

$>$ Gekappt<, >konstitutiv unerreichbar $<-$ wenn es zutrifft, dass die »ästhetische Dimension « für Leichte Sprache prinzipiell unzugänglich ist, ist auch die Literaturtauglichkeit von Leichter Sprache grundsätzlich in Frage gestellt und diese wie ihre Leser faktisch auf den Bereich der »Standardwelt« (im Sinne von Adamzik 2004, vgl. Fix 2017, S. 170 f.) eingegrenzt. Diesem, in seinen Voraussetzungen noch genauer zu diskutierenden, skeptischen Befund von Bredel/Maaß (2016) steht mindestens in der Theorie der Teilhabegedanke entgegen, der nach der »UN-Behindertenrechtskonvention « (die seit ihrem Beschluss 2006 maßgeblich zur Durchsetzung von Leichter Sprache in der Öffentlichkeit beigetragen hat) ausdrücklich auch die »Teilhabe am kulturellen Leben « und das Recht auf »Zugang von Menschen mit Behinderungen zu kulturellem Material« umfasst (Art. 30 (1) a) (Übereinkommen 2017, S. 26). Aber auch in der Praxis zeigt sich eine stetig wachsende Zahl an Übersetzungen (oder auch: Vereinfachungen, Köster 2018) von literarischen Texten in vereinfachte Sprache und original verfassten literarischen Texten, die z.Zt. vor allem noch in spezialisierten Verlagen wie dem Passanten- oder dem Spaß am Lesen-Verlag oder in eigenen Reihen wie Einfach klassisch im Schulbuch-Verlag Cornelsen erscheinen. Insbesondere die in den letzten Jahren, zunächst durch Literaturwettbewerbe angeregte original in vereinfachter Sprache verfasste Literatur (vgl. Lebenshilfe 2015, 2017; Lüthen 2019, S. 7) steht dabei noch im Zeichen des Neuen und Experimentellen: »Aufbruch in eine andere Literatur « schrieb sich das renommierte Literaturhaus Frankfurt für sein »Pionier-Projekt« Literatur in Einfacher Sprache auf die Fahnen (Literaturhaus 2019), an dem sich bekannte Schriftsteller wie Alissa Walser, Julia Schoch oder Arno Geiger beteiligten. Mit den mittlerweile in Buchform vorliegenden Geschichten »bekommt die Literaturgeschichte ein neues Kapitel«, so ihr Herausgeber Hauke Hückstädt (Hückstädt 2020, S. 257).

Das umrissene neue literarische Feld von Originaltexten und Übersetzungen bzw. Vereinfachungen ist nicht ausschließlich den Regelwerken durchnormierter Leichter Sprache verpflichtet, sondern orientiert sich häufig an den etwas weniger strikt geregelten (vgl. Bredel/Maaß 2016, S. 527 ff.) Leitlinien »Einfacher Sprache«. Die Grundsatzfrage, ob bzw. welche Grenzen der Literarästhetik in Leichter und/oder Einfacher Sprache verfasster Texte gesetzt sind, stellt sich für verschiedene Ausprägungen vereinfachter Sprache gleichermaßen.

Während - wie oben knapp dokumentiert wurde - in der Linguistik langsam ein Forschungsfeld zu Leichter Sprache entsteht, scheint eine literaturwissenschaftliche Auseinandersetzung mit dem Gegenstand bislang noch weitgehend ein Desiderat zu 
sein (siehe allerdings jetzt Wisotzki 2021). Anregungen zur Auseinandersetzung mit literarischer Einfachheit und einem produktiven Umgang mit ihr kommen allerdings aus der Kinder- und Jugendliteraturforschung (Lypp 1984; O’Sullivan 2016) und aus der Literaturdidaktik. In letzterer spielt der Aspekt der Vereinfachung sowie des Verhältnisses von Einfachheit und literarischer Ästhetik besonders da eine Rolle, wo es um das Für und Wider der Vereinfachung kanonischer Texte der literarischen Tradition geht. So plädiert Cornelia Rosebrock für den Einsatz »[v]ereinfachte[r] Klassikerausgaben für den Schulgebrauch«, ohne das »anspruchsvolle Ziel« der Vermittlung von »Befremdung und Nicht-Verstehen als Bestandteil ästhetischer Erfahrung « aus den Augen zu verlieren (2015, S. 38; vgl. die Bestandsaufnahme bei Köster 2018; Mesch 2020). Daneben beschäftigt die Literaturdidaktik seit längerem die Frage, inwieweit mit Rosebrock (2019) textseitige >Textkomplexität< und leserseitig bedingte >Textschwierigkeit< von Literatur zu bestimmen und in der Literaturvermittlung zu berücksichtigen sind (vgl. zum aktuellen Forschungsfeld der >Textpassung< Frickel 2018). Durch den aktuell zu beobachtenden starken Ausbau einer Produktion von literarischen Texten in Einfacher, teilweise sogar Leichter Sprache erhält die Frage nach der Vereinbarkeit von Literarästhetik und Einfachheit nun erhöhte, über Kontexte der genannten Art hinausgehende Aufmerksamkeit.

Der vorliegende Beitrag möchte unter diesen Voraussetzungen eine Auseinandersetzung mit der Frage nach den Möglichkeiten und Grenzen von Literatur und Literarästhetik in vereinfachter Sprache an der Schnittstelle von Linguistik und Literaturwissenschaft anstoßen. Wir möchten zeigen, dass auch Texten in vereinfachter Sprache und ihren Leserinnen und Lesern die literarästhetische Dimension nicht verschlossen ist. Einfachheit und Ästhetik schließen sich nicht aus, sondern stehen, wie auch die grundlegenden Parameter von Einfachheit und Komplexität, in einem skalierten Verhältnis des Mehr oder Weniger zueinander. Wie sich diese Annahmen begründen und beispielhaft belegen lassen, ist der Gegenstand der folgenden Überlegungen. Wir beschäftigen uns zunächst damit, was unter > Literatur $<$ und ihrer ästhetischen Dimension zu verstehen ist (2.). Im Anschluss daran setzen wir uns mit den Vorstellungen von Einfachheit auseinander, wie sie in den bestehenden Regelwerken zu Leichter und Einfacher Sprache (3.), in der gegenwärtigen Linguistik (4.) und Literaturwissenschaft (5.) vertreten werden. Hieraus lässt sich ein erstes theoretisches Fazit zur Literarästhetik von Literatur in vereinfachter Sprache ableiten, das wir am Ende von Abschnitt (5.) vorstellen. In den beiden letzten Abschnitten konfrontieren wir unsere theoretischen Überlegungen mit der Praxis vorliegender Regeln und Empfehlungen zum Verfassen von Literatur in vereinfachter Sprache (6.), die wir schließlich um einige wenige exemplarische Beobachtungen an drei literarischen Beispielen ergänzen (7.).

\section{Literaturbegriff und ästhetische Dimension des Textes}

Wie die zitierte, kritische Einschätzung der Möglichkeit von >Literatur < unter den Bedingungen Leichter oder Einfacher Sprache durch Bredel/Maaß indirekt verdeutlicht (plakativ Baumert 2016, S. 206: »Es geht nicht.«), setzt die Auseinandersetzung mit der Frage nach der Literaturfähigkeit von Leichter und Einfacher Sprache zu- 
nächst eine Verständigung darüber voraus, was unter >Literatur $<$ bzw. genauer, wie Bredel/Maaß formulieren, unter der >ästhetischen Dimension von Texten $<$ verstanden werden soll.

Folgt man zunächst der deskriptiven Minimaldefinition von >Literatur $<$ im Sinne der empirischen Literaturwissenschaft als einem >gesellschaftlichen Handlungssystem $<$, dann umfasst Literatur alle Handlungen eines Akteurs »mit einem oder in bezug auf ein sprachliches Gebilde, das er nach seinen Vorstellungen für literarisch hält, anderen als literarisch anbietet bzw. als literarisch bewertet.« (Hauptmeier/ Schmidt 1985, S. 15; vgl. Jacob 2016). Es gibt demnach Literatur auch in vereinfachter Sprache, sobald sich erfolgreiche Handlungen in Bezug auf ihre Herstellung, Rezeption und Vermittlung im Handlungssystem Literatur identifizieren lassen. Als solche könnte etwa die bereits erwähnte Frankfurter Anthologie Literatur in Einfacher Sprache (Hückstädt 2020) gelten, die im renommierten deutschen Publikumsverlag Piper erschienen ist, dort aufwendig präsentiert und wohlwollend von einigen deutschen Leitmedien aufgenommen wurde (Piper 2020). Ein anderes Beispiel ist das Themenheft Qualität (!) des Magazins brand eins, das im Herbst 2019 unter der Ankündigung: »Die Leichte Sprache nimmt den Inhalt ernst, aber nicht schwer«, die Szene »Nacht« aus Johann Wolfgang Goethes Faust I in einer Interlinearübersetzung in Leichter Sprache anbietet (Goethe/Fröhlich 2019). Empirisch wäre damit die Frage, ob Leichte Sprache literaturfähig ist, bereits positiv beantwortet. Zu beobachten wäre allerdings im Anschluss, ob diese und andere Selbstzuschreibungen von Autor, Redaktion und Verlag im gesellschaftlichen Handlungssystem Literatur dauerhaft überzeugend, d.h. gesellschaftlich akzeptiert sind oder nicht.

Ursula Bredel und Christiane Maaß bestreiten nun jedoch auch nicht die Existenz von Literatur in Leichter Sprache - die von Maßß geleitete Forschungsstelle Leichte Sprache an der Universität Hildesheim hat sich vielmehr 2014 bis 2016 selbst auch für die Übersetzung Grimmscher Märchen engagiert (Forschungsstelle Leichte Sprache 2020; NDR2020) -, Bredel/Maaß konstatieren aber, wie oben zitiert, dass sich in ihr die »ästhetische Dimension von Texten« nicht erreichen lasse bzw. der Herstellung von »literarästhetischen Texten in Leichter Sprache enge Grenzen gesetzt « seien (Bredel/Maß, S. 518f.). Damit kommt die Frage eines engeren, hier als >ästhetisch < qualifizierten Literaturbegriffs ins Spiel, nach Klaus Weimars Begriffsexplikation im »Reallexikon der deutschen Literaturwissenschaft « die Frage danach, was die >Literarizität< oder >Poetizität< eines Textes ausmacht (Weimar 2000, S. 443). Bredel/Maaß bestimmen Literarizität über eine offene Merkmalsreihe, zu der Mehrdeutigkeit, Variantenreichtum im sprachlichen Ausdruck, Aufbrechen von Logik und Chronologie sowie Selbstbezüglichkeit gehören, die im Ganzen als »ein Experimentieren mit Sprache« charakterisiert ist (Bredel/Maaß 2016, S. 519). Weimar setzt demgegenüber in seiner Definition zunächst nicht bei besonderen Textmerkmalen an, sondern bei der spezifischen Wahrnehmung von >literarischen< Texten, die sich darin als solche erweisen, dass ihre Rezipienten

ihnen gegenüber eine besondere Lese-Einstellung (Rezeptionshaltung) einnehmen: Man suspendiert die Referenz und läßt dahingestellt sein, ob auch stimmt (zutrifft, jemals der Fall war), was man liest. (Weimar 2000, S. 443 f.) 
Einen Text als >literarischen< Text wahrnehmen heißt demnach zuerst, ihn nicht als Aussage über einen Sachverhalt in der realen Welt, sondern bezogen auf eine vorgestellte Welt zu verstehen, deren Wirklichkeitsreferenz >dahingestellt< bleiben kann. Damit ist indirekt bereits das Kriterium der Fiktionalität eingeführt, das häufig zur Definition von Literatur herangezogen wird, zugleich jedoch die grundlegende kognitive Bedingung auf Seiten der Rezipienten benannt, die erfüllt sein muss, um einen Text als Literatur wahrnehmen zu können. Anders formuliert: die Fähigkeit, mit einem Text anderes als reine Informationsentnahme verbinden zu können. Im Umkehrschluss deutet sich hier jedoch auch schon an, dass die basale Erfahrung, die ein literarischer Text stiften kann, darin liegt, »eine eigene Wirklichkeitsvorstellung zu erzeugen« (Stefan Matuschek, zit. nach Köster 2018, S. 65) und darin auch die, teils gezielt eingesetzte, Unbestimmtheit seiner sprachlichen Abstraktheit zu konkretisieren (Ingarden 1997 [1968], §§7-14). Beides schließt sowohl an die Wirklichkeitserfahrung der Lesenden als auch an ihr Sprachwissen an und wird in der temporären Loslösung vom >Zwang< der Referenz zugleich überstiegen und erweitert. Es ist leicht zu sehen, dass sich diese elementare literarische Erfahrung auch an Texten vereinfachter Sprache machen lässt und von der individuellen Textgestalt und Rezeptionskompetenz abhängig ist.

Ausgelöst wird diese ästhetische Leseeinstellung nach Weimar zum Beispiel - entsprechendes Textsortenwissen vorausgesetzt - durch »Selbstklassifikationen « (Weimar 2000, S. 444) von Texten als >Roman<, >Ballade< oder >Märchen<. Unabhängig davon wird sie veranlasst, »wenn etwas an einem Text sich nicht verträgt mit [alltagssprachlich, konventionell] Vorausgesetztem«(Weimar 2000, S. 444.), d.h. (um einen weiteren einschlägigen Begriff zur Bestimmung von Literatur ins Spiel zu bringen) wenn Verfremdungseffekte wahrgenommen werden. Dies gilt, wie Weimar anführt, bereits für den künstlichen Zeilenumbruch in einem Gedicht im Unterschied zum üblichen seitenfüllenden Satzspiegel, für den Einsatz von Reimen, sprechenden Tieren oder eines allwissenden Erzählers. So lässt sich zusammenfassen:

Als literarische Texte qualifizieren sich demnach solche, die nachweislich Anlaß bieten, die übliche Lese-Einstellung abzulösen durch eine andere, und die sich dazu anbieten, als Gegenstand auch zu anderen Zwecken als zu dem der Information über anderes in Gebrauch genommen zu werden [...]. (Weimar 2000, S. 444)

Dieser einerseits epistemologisch genaue, andererseits aber formal weitgehend offene Definitionsvorschlag für >Literatur < hat zunächst den Vorteil, dass das Kriterium für Literarizität nicht ausschließlich in der Textgestalt, sondern auch in ihrem Gebrauch verankert ist, d.h. in einem relativen Zusammenspiel von Text und Rezipient, das über die reine Informationsentnahme hinausgeht. In diesem Sinne können dann z.B. auch alteingeführte, wenn auch im Bildungsdiskurs nicht immer hochgeschätzte ästhetische Merkmale wie Freude, Spaß und Genuss an Texten rehabilitiert werden, auf die vielleicht nicht zufällig der auf Literatur in vereinfachter Sprache spezialisierte »Spaß am Lesen«-Verlag schon in seinem Verlagsnamen rekurriert. Freude und Spaß könnten allerdings auch, so Ulla Fix in ihren Überlegungen da$\mathrm{zu}$, wieviel sprachliche >Schwere< und >Zumutungen< Texte in Leichter Sprache vertragen können, von der »`Entzifferung< der unüblich präsentierten Zeichenge- 
stalt « ästhetischer, literarischer Texte ausgehen (Fix 2017, S. 172; zur Differenz von >Schwierigkeit< in Sachtexten und literarischen Texten siehe auch Rosebrock 2019). Für die Bestimmung des literarästhetischen Potentials von Texten in Leichter Sprache ist demnach nicht nur nach der Realisierung bestimmter als >ästhetisch< angesetzter Textmerkmale zu suchen, sondern auch ihr Vermögen, die Bereitschaft für sprachliches Spiel, Variation oder Tolerierung von Inkonsistenz und Unwahrscheinlichkeit oder auch für den Möglichkeitssinn als Merkmale einer ästhetischen Einstellung zu einem Text zu wecken, zu ermutigen oder zu verstärken.

Ungeachtet dieser notwendigen rezeptionsästhetischen Erweiterung der Betrachtungsperspektive bleibt die These zu prüfen, dass »die Realisierung der poetischen Sprachfunktion (Jakobson) mit Leichter Sprache konstitutiv unerreichbar « sei (Bredel/Maaß 2016, S. 519). Roman Jakobsons breit rezipierte und ungenannt auch in Weimars Literaturdefinition eingegangene Bestimmung der >poetischen Sprachfunktion< umfasst alles, was in der Wahrnehmung des Wortes »nicht bloß als Repräsentant des benannten Objekts [...] empfunden wird « (Jakobson ${ }^{3} 1993 \mathrm{a}$, S. 79), sondern als Wort in irgendeiner Form Eigenwert gewinnt. Das klassische Beispiel hierfür ist der Reim, der durch seine Lautgestalt zwei Worte unabhängig von ihrer Bedeutung miteinander verbindet. Ist der Reim damit ein literarästhetisches Mittel, das sich zweifellos schon durch einfachste Kinderverse belegt generell auch vereinfachter Sprache anbietet, gilt dies nicht weniger für die Klanggestalt der Sprache überhaupt, die eine eigene, sinnlich erlebbare, d.h. im Wortsinn >ästhetische<, Dimension darstellt. Ist auch diese vereinfachter Sprache notwendig eigen, lässt sich daraus umgekehrt aber auch, wo es um Bewertung geht, eine Forderung an die literarästhetische Dimension vereinfachter Sprache ableiten: dass sie gut (interessant, schön) klingen soll. - In diesem Zusammenhang ist auch auf die allgemein lange in der Forschung vernachlässigte Bedeutung des Vorlesens literarischer Texte hinzuweisen, die im Kontext vereinfachter Sprache mit Blick auf die Adressatengruppen nicht nur pragmatisch von Bedeutung ist, um verminderte Lesefähigkeit zu kompensieren, sondern auch als eine eigenständige Gestaltungsebene hervortreten kann, die bspw. die stilistische schriftsprachliche Monotonie einer Folge von einfachen Hauptsätzen auflöst: »Beim [Vor-]Lesen der Texte verschwindet das Regelwerk noch weiter ins Unhörbare.« (Lüthen 2019, S. 44, vgl. auch S. 41-44; Gernet 2017, S. 21 f.)

Auf der Bedeutungsebene des sprachlichen Zeichens realisiert sich die poetische Funktion durch sprachlich-rhetorische Mittel wie auffallende Wortfügungen und Wortstellungen, Metaphern u.a., soweit diesen kein unmittelbar informativer Wert zukommt. Mit den Normen vereinfachter Sprache kollidieren diese Mittel, insofern sie die Verständlichkeit des Textes auf der Ebene des Literalsinns erschweren können - aber sie darum vollständig aus Texten Leichter (oder gar Einfacher) Sprache mit literarästhetischem Anspruch auszuscheiden, ist nicht plausibel.

Die Auffassung, dass die poetische Sprachfunktion nach Jakobson mit Leichter Sprache oder auch per se das Experimentieren mit Sprache nicht realisierbar ist, ist darum in dieser Schärfe nicht zu halten (zumal, wenn man mit Jakobson davon ausgeht, dass die poetische Sprachfunktion immer auch schon in der Alltagssprache wirksam ist, Jakobson ${ }^{3} 1993$ b, S. 92 f.). Die von uns unten unter (7.) diskutierten Beispiele für realisierte Literarästhetik in Leichter und Einfacher Sprache sollen dies auch für die literarische Praxis belegen. Zunächst aber soll es darum gehen, was 
$>$ leicht $<$ und >einfach $<$ in linguistischer und literaturwissenschaftlicher Perspektive ist.

\section{Was ist >leicht< an Leichter Sprache? Die Regelwerke}

Für eine Annäherung an die Frage, was eigentlich leicht an Leichter Sprache ist, werfen wir zunächst einen Blick auf die Regelwerke. Die meisten Regelwerke Leichter Sprache sind aus der Praxis entstanden: Das »Netzwerk Leichte Sprache « ist ein »Zusammenschluss von Akteuren aus dem Bereich der Arbeit mit Personen mit geistiger Behinderung gegründet« (Bredel/Maaß 2016, S. 83). Ihr Regelwerk hat das Netzwerk 2009 auf seiner Homepage veröffentlicht. Die Regeln von Inclusion Europe wurden ebenfalls 2009 veröffentlicht. Sie sind im Kontext eines Projekts entstanden, »das sich dem Thema Erwachsenenbildung für Personen mit geistiger Behinderung verschrieben hat (Bredel/Maßß 2016, S. 84). Die »Barrierefreie-Informationstechnik-Verordnung (BITV 2.0) « schließlich trat 2011 in »Umsetzung der in der UN-Behindertenrechtskonvention geforderten Grundsätze « in Kraft (Bredel/ Maаß 2016, S. 82). Bemerkenswert ist: »Bei keinem der drei Regelwerke waren Sprach- oder Übersetzungswissenschaftler in nennenswerter Form an der Genese beteiligt.« (Bredel/Maßß 2016, S. 82) Daraus können wir schlussfolgern: Die durch die Regelwerke manifestierten Vorstellungen davon, was Einfachheit ist, beruhen auf laienlinguistischer Intuition. Gerd Antos, der »Vater « der Laienlinguistik, rekonstruiert in einem Beitrag zu Leichter Sprache als Politolekt, was Einfachheit - hier »Verständlichkeit« - für Laien bedeutet. Laut ihm verbinden sie damit u.a. sprachlich bzw. kommunikativ Vertrautes, emotional Ansprechendes, subjektiv Einleuchtendes, Prägnantes, unmittelbar Schlüssiges und leicht Behaltbares (Antos 2017, S. 138). Den Leichte-Sprache-Regelwerken können wir entnehmen, was die Konsequenzen eines solchen laienlinguistischen Globalverständnisses von Verständlichkeit für die Modellierung verständlicher resp. Leichter Sprache sind. Laut der Regelwerksynopse von Bredel/Maaß (2016. S. 109ff.) beziehen sich die Regeln auf die folgenden Bereiche: Mediale und visuelle Gestaltung, Schriftzeichen, Morphologie, Lexik, Syntax, Semantik, Text. Wir beziehen uns in diesem Beitrag ausschließlich auf die Bereiche Morphologie, Syntax, Semantik und Text. Deshalb sei der Synopse entnommen, was Gegenstand der Regeln in diesen Bereichen ist:

- Morphologie: Lange Komposita, Präteritum, Konjunktiv, Genus/Gendern, Genitiv, Passiv;

- Syntax: Umfang der Sätze, Nominalstil, Fokusstrukturen/Inversion, Konnektoren;

- Semantik: Vermeidung von Negation; nur eine Aussage pro Satz;

- Text: klare Benennung von Textthema und Textfunktion, Beschränkung der Information, Konsistenz der Bezeichnung, pronominale Wiederaufnahme, thematische Entfaltung, Redundanz, Fußnoten, Glossare, Zwischenüberschriften, Adressierung.

Für die Ebene der Morphologie lässt sich ein Begriffsverständnis rekonstruieren, das unter leichter/einfacher/verständlicher Sprache eine Sprache versteht, die kurze Wörter verwendet und auf einen Teil der im Deutschen vorhandenen morphologi- 
schen Kategorien verzichtet. Als syntaktisch einfach werden kurze, verbalstilistische Sätze verstanden. Dem Verständnis von kurzen Sätzen auf syntaktischer Ebene entspricht das semantische Postulat »nur eine Aussage pro Satz« - ein klares Verdikt zur Nebensatzvermeidung. Als textuell einfach können nach diesem Verständnis Texte gelten, die den Leser linear durch eine einfach strukturierte Entfaltung eines klar benannten, auf bestimmte Informationsbereiche beschränkten Themas führen.

Aufschlussreich ist, wie die linguistische Forschung mit dem Regelwerkverständnis von Leichter Sprache umgeht. Christiane Maaß sieht - nicht zuletzt vor dem Hintergrund ihrer übersetzungswissenschaftlichen Perspektive - die Aufgabe der Linguistik darin, das Regelwerk auf eine linguistisch fundierte Basis zu stellen. Das Ergebnis ist das 2015 veröffentlichte »Leichte Sprache. Das Regelbuch«, »das sich explizit an Übersetzerinnen und Übersetzer von Leichte-Sprache-Texten richtet und die [...] öffentlich zugänglichen Regelwerke kritisch reflektiert« (Bredel/Maaß 2016, S. 82). Maaß formuliert die folgenden »Grundprinzipien« mit Bezug auf das Sprachsystem (2015, S. 76):

1. Grammatische Funktionen mit eigenem Träger ausstatten (Negation, Vergangenheitsformen, Genitiv: eigene Funktionsmarker); nicht: »er aß« sondern: »er hat gegessen $«$

2. Zentral statt peripher (Wortschatz, Informationsverteilung, Genitiv, Konjunktiv)

3. Handlungsorientierung (verbal statt nominal, Handlungsträger benennen, Informationsverteilung)

4. Wichtiges und Zentrales mehrfach hervorheben (»Redundanzprinzip«, »Multicodalität«)

Einen völlig anderen Weg geht Bettina Bock (2018), indem sie die Veröffentlichung von Ergebnissen aus dem o.g. Leipziger Projekt mit dem Titel versieht » Leichte Sprache < - Kein Regelwerk«. Der regelpositivistischen Herangehensweise von Maßß setzt sie damit die Auffassung entgegen, dass gegenüber einem starren Regelwerk - so fundiert auch immer es begründet sein mag - allgemeine Verständlichkeitsprinzipien zu bevorzugen seien. Sie begründet das wie folgt:

Wir gehen allerdings davon aus, dass Regellisten nicht ausreichen, um Textqualität zu sichern. [...] Wir schlagen also vor, »Leichte Sprache« nicht mehr in dieser strikten Form an Regeln gebunden zu verstehen. Stattdessen definieren wir sie wesentlich über ihre Intention bzw. ihre Funktion: »Leichte Sprache« dient dazu, Kommunikation für Personenkreise verständlich zu machen und barrierefrei aufzubereiten, die sonst von dieser Kommunikation ausgeschlossen wären. (Bock 2018, S. 11)

Bock plädiert folglich dafür, »die existierenden Regelwerke eher als Sammlungen von Faustregeln zu verstehen und nicht als strikte Gesetze« (2018, S. 15). Als flexibleren Rahmen für Bemühungen um eine verständliche und barrierefreie Kommunikation präsentiert sie fünf Angemessenheitsfaktoren. Es geht folglich um die Frage: 
Ist der Text angemessen in Bezug auf...?

1. den Adressaten bzw. den tatsächlichen Leser [...]

2. die Textfunktion [...]

3. den Inhalt bzw. den thematisierten Gegenstand [...]

4. die Situation [...]

5. den Sender [...] (Bock 2018, S. 16)

Mit einer Orientierung an allgemeinen Verständlichkeitskriterien folgt Bock einer Stoßrichtung, die im Sammelband Bock/Fix/Lange (2017) u.a. auch von Ulla Fix aus textlinguistischer und von Ursula Christmann aus psycholinguistischer Perspektive eingeschlagen wird. Mit der Aufnahme der Textfunktion als Angemessenheitskriterium bietet sich Spielraum für unsere Fragestellung: In diesem Sinne hätten literarische Texte in Leichter Sprache nicht nur den durch die Perspektive der Adressierung gegebenen Einfachheitskriterien zu genügen, sondern wären im Sinne von Adamzik einer »Textwelt« verpflichtet, die sich abweichend von der »Standardwelt « (siehe auch oben, 2.) als »Welt des Spiels, der Fantasie« entfaltet (vgl. Fix 2017, S. 170f.).

Sicherheitshalber sei an dieser Stelle betont: Es geht hier nicht darum, die Maaß'sche und Bock'sche Herangehensweise gegeneinander auszuspielen. Das auf das Leipziger Projekt zurückgehende Plädoyer für einen flexibleren Zugang mit systematischem Rückgriff auf Textlinguistik und Verständlichkeitsforschung ist aus linguistischer Perspektive sehr attraktiv. Andererseits muss damit gerechnet werden, dass die Praxis weiterhin Bedürfnisse nach klar umrissenen Regeln generieren wird (die ihre Bedeutung auch für die literarische Textgenese, mindestens bei der Transformation in Leichte Sprache, behalten werden).

\section{Einfachheit aus linguistischer Perspektive}

Im Fokus der vereinfachten Sprache steht das Ziel sprachlicher Einfachheit, im Hinblick auf die Literatur geht es um Formen »einfacher und vereinfachter literarischer Texte «, wie Juliane Köster zusammenfasst (2018, S. 58). Doch was ist Einfachheit? Für eine linguistische Bestimmung von Einfachheit müssen wir den >Umweg< über die Komplexität gehen: Während Komplexität gerade in jüngerer Zeit ein zentraler Gegenstand linguistischer Forschung war und ist (vgl. die Sammelbände Sampson/Gil/Trudgill 2009, Kortmann/Szmrecsanyi 2012 sowie Hennig 2017), ist Einfachheit kein Gegenstand (system-)linguistischer Diskussion. Dieses Ungleichgewicht der linguistischen Aufmerksamkeit liegt sicherlich daran, dass komplexere Strukturen die größere Herausforderung, das interessantere Betätigungsfeld für die linguistische Beschreibung und Theoriebildung sind. Auf der anderen Seite spielt die Frage nach Einfachheit eine zentrale Rolle in der linguistischen Verständlichkeitsforschung (vgl. Christmann 2017). Trotz der starken Heterogenität konkurrierender Komplexitätsbegriffe kann die Skalarität des Komplexitätsbegriffs als grundlegende Annahme betrachtet werden. Wenn Komplexität der Pol einer Skala ist, ist Simplizität der Gegenpol. Insofern erscheint es uns legitim, bei dem Versuch, den Begriff der Einfachheit linguistisch zu bestimmen, auf die linguistische Komplexitätsdiskussion zurückzugreifen. 
Abb. 1 Parameter von Komplexität/Simplizität (Zeman 2017, S. 55)

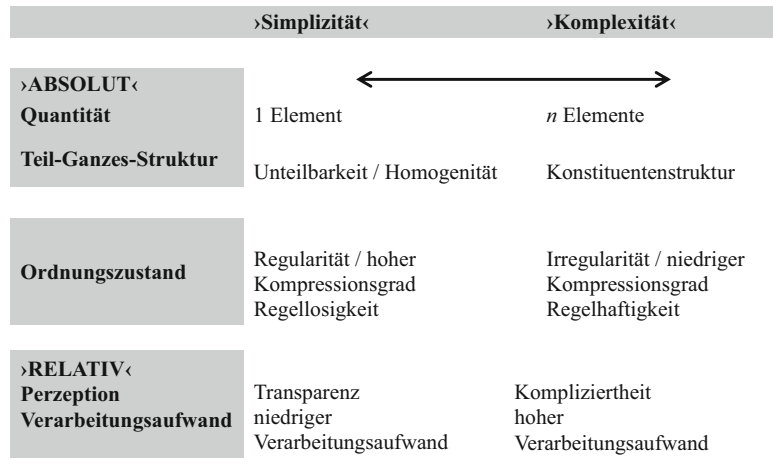

Sonja Zeman (2017) legt ein Komplexitätsmodell vor, das Simplizität als Gegenpol bereits mit einbezieht (s. Abb. 1).

Mit der Unterscheidung zwischen absoluter und relativer Komplexität rekurriert Zeman auf einen zentralen Parameter der Komplexitätslinguistik. Die Unterscheidung geht auf Miestamo (2008) zurück. Laut Miestamo ist absolute Komplexität aus theoriegeleiteter, objektiver Perspektive zu definieren als »number of parts in a system «, während relative Komplexität aus nutzerorientierter Perspektive zu bestimmen ist »in terms of costs and difficulty to language users « (Miestamo 2008, S. 24). Fischer bezeichnet deshalb die absolute Komplexität als »Beobachterkomplexität « und die relative Komplexität als »Benutzerkomplexität« (Fischer 2017, S. 22). Absolute Komplexität oder Simplizität ist also das Ergebnis der Beobachtung sprachlicher Äußerungen oder auch sprachlicher Systeme durch den Linguisten. Mit >Ordnungszustand < bringt Zeman neben der Unterscheidung zwischen absoluter und relativer Komplexität eine dritte Dimension in die Modellierung der Skala ein. Es geht ihr hier um das Verhältnis von idiosynkratischen, also nicht weiter zerlegbaren Formen - vgl. bspw. das in drei Teile zerlegbare Wort hahaha oder das nicht weiter zerlegbare Wort komplex: Hier kann man nun je nach Perspektive die idiosynkratische Form komplex als komplexer betrachten, weil sie nicht auf einer regulären kompressierbaren Struktur basiert, oder aber als weniger komplex, da sie nicht auf Musterbildung beruht und somit keine Regeln zu ihrer Erzeugung angewendet werden müssen (vgl. Zeman 2017, S. 54). Gerade für die Diskussion von Fragen nach Zusammenhängen von Komplexität und Literarästhetik ist die Frage nach der Bewertung von Ordnungszuständen natürlich höchst relevant, aber gerade darum auch ein eigenes Thema, das wir im Abschnitt 7 am Beispiel von Herrndorfs Tschick nur exemplarisch andeuten können. Denn auch literarästhetisch ist die Frage der Komplexität von Ordnungs- bzw. Unordnungszuständen, regelfolgenden bzw. regelverletztenden Arrangements als jeweils komplex(er) eine Frage der Perspektive bzw. des Ansatzes. Hinzukommt die zusätzliche Relation von literarischem Artefakt und natürlichem Sprachsystem, innerhalb derer Ordnungsmuster affirmiert, ganz oder teilweise verletzt und bzw. oder durch eigene literarische ersetzt werden können. Noch einmal hiervon zu unterscheiden ist schließlich die für komplex oder weniger komplex gehaltene Anforderung, Ordnung, Unordnung, Regelbefolgung oder Regelverletzung in der literarischen Rezeption wahrzunehmen. So stellen beispiels- 
weise nach Maria Lypp Rhythmus und Klang im Kindervers als »übersprachliche Strukturierung, die als Zusatz zur sprachlichen Strukturierung einen Zuwachs an Komplexität ausmacht«, für Kinder gerade nicht eine komplexere »höhere Anforderung « in der Rezeption dar, insofern die »geringe Zahl an Regeln, nach denen der Kindervers strukturiert ist, [...] gegenüber der Vielzahl der Regularitäten des Sprachmaterials, das in ihm verwendet wird, eine Vereinfachung dar[stellt]«, mit der dem Kind »die Regelkomplexität der natürlichen Sprache subjektiv beherrschbar zu werden scheint.« (Lypp 1984, S. 31)

Für unseren Kontext ist aber auch die Unterscheidung zwischen absoluter und relativer Komplexität höchst relevant. Relative Komplexität repräsentiert die Angemessenheitsdimension >Adressat< im Bock'schen Modell (s.o.). Da Adressierung konstitutiv für Leichte Sprache ist, ist die Verständlichkeitsdimension der Simplizitäts-Komplexitäts-Skala von zentraler Bedeutung. In der Praxis materialisiert sich die Relevanz dieser Dimension in der Direktive des Prüfens (vgl. Bredel/Maaß 2016, S. 107); in der Forschung spiegelt sich die Wichtigkeit der relativen Komplexität/ Einfachheit in der methodischen Berücksichtigung der Rezeptionsseite und in der Forderung nach und Durchführung von empirischen Untersuchungen wider (s.o.). Und für diese wäre schließlich nach den unter (2.) dargelegten Kriterien des Literarischen nicht nur danach zu fragen, wieviel Komplexität in einem Text verstanden, sondern auch, welches Maß an Komplexität er in der Vorstellungswelt von Lesern anregen kann.

Das bedeutet aber umgekehrt nicht, dass wir auf den Begriff absoluter Komplexität/Einfachheit verzichten können. Diese Dimension repräsentiert den analytischen Zugang, bei dem es darum geht, zu erfassen, was überhaupt sprachliche Systemeigenschaften und Strukturen zu einfachen oder komplexen Systemeigenschaften und Strukturen macht.

Mit dem Verweis auf Systemeigenschaften und Strukturen beziehen wir uns auf eine zweite zentrale Unterscheidung der Komplexitätslinguistik. Fischer (2017) unterscheidet - mit Rückgriff auf Dahl (2004) - zwischen Systemkomplexität und struktureller Komplexität. Systemkomplexität meint die Komplexität der Ressourcen und Regularitäten, strukturelle Komplexität die Type-Komplexität der Ausdrücke und die Token-Komplexität von Äußerungen/Texten (Fischer 2017, S. 23). Indem in den Regelwerken bestimmte grammatische Kategorien ausgeschlossen werden, wird angenommen, dass bestimmte Bestandteile des Sprachsystems eine zu hohe Komplexität für die Zielgruppe aufweisen. Andererseits geht es immer um konkrete Texte und damit darum, dass die strukturelle Komplexität sprachlicher Äußerungen so gering wie möglich gehalten werden soll.

Doch zurück zu Zemans Modellierung. Mit der Komplexitätsdimension Quantität bezieht sie sich ebenfalls auf eine Kerngröße der Komplexitätsdiskussion. Bei aller Heterogenität der Ansätze und Komplexitätsbegriffe kann doch als gemeinsamer Nenner ausgemacht werden, dass es bei Komplexität immer um ein »Mehr an« geht. Fischer (2017) nimmt das »Mehr an « in die Begriffsbestimmung von Komplexität auf, indem er den Beschreibungsaufwand zum Gradmesser für die Bestimmung von Komplexität macht: »X ist komplexer als $\mathrm{y}$, wenn die kürzeste Beschreibung der Regularitäten oder strukturierten Muster von x länger ist als die kürzeste Beschreibung der Regularitäten oder strukturierten Muster von y.«(Fischer 2017, S. 22) 
Pohl benennt »Komplexität als Menge«, d.h. »Komplexität durch Addition« als eine Komplexitätsdimension (Pohl 2017, S. 253), der er die Komplexitätsdimensionen »Komplexität als Unterschiede« (Substitution) und »Komplexität als Einbettung« (Integration) an die Seite stellt. Er begrenzt sich für sein Anliegen (Komplexität im Bereich konzeptioneller Schriftlichkeit im Unterrichtsdiskurs) sogar auf Komplexität durch Addition. Und in der Tat liegt darin eine gewisse Attraktivität, denn man kann diesen Begriff beliebig - wie Pohl es formuliert - »konzeptuell flankier[en] und damit näherhin profilier[en« (Pohl 2017, S. 253). Mit anderen Worten: Ganz im Sinne auch der Abbildung der Komplexitätsdimension Quantität im Modell von Zeman geht es also zunächst einfach um skalare Übergänge zwischen Anzahlen an Elementen. Was als ein solches Element zu gelten hat, ist völlig offen. So kann dieser Komplexitätsbegriff sowohl in Verbindung gebracht werden mit systembezogener als auch struktureller Komplexität. Er kann auf beliebige linguistische Beschreibungsebenen angewendet werden, also folglich auch als Folie für eine Bewertung der in den Leichte-Sprache-Regelwerken bearbeiteten Bereiche genutzt werden. Darüber hinaus ist dieser Begriff - und das macht ihn attraktiv für unser Anliegen - genauso gut auf andere Felder übertragbar. Somit können wir hier die Frage stellen, ob es literarästhetische Simplizität bzw. Komplexität gibt, die sich ebenfalls durch ein Mehr oder Weniger an bestimmten literarästhetischen Merkmalen ausmachen lässt (s.u.).

Ein quantitätsbezogener Komplexitäts- oder Simplizitätsbegriff hat den Vorteil, dass er nicht nur Flexibilität in Bezug auf den Gegenstand erlaubt, sondern auch flexibel in Relation zur relativen Komplexität/Simplizität zu bringen ist: Bekanntlich ist etwas, was für einen Rezipienten einfach oder komplex ist, nicht automatisch auch einfach oder schwer für einen anderen (vgl. auch Imo/Lanwer 2017: »Sprache ist komplex. Nur: Für wen?«). Auf diese Weise können Kerngrößen der Simplizität/ Komplexität für einzelne Rezipienten oder Rezipientengruppen individuell bestimmt werden. Aus dieser Perspektive kann aber auch als Grundproblem der LeichteSprache-Regelwerke benannt werden, dass diese Einfachheit/Komplexität qualitativ festschreibt. Dabei ist - so Zeman (2017) - »Komplexität sowohl als ontologische Kategorie als auch als Kategorie der Beobachtung ein relationales Konzept. [...] Der Umstand, dass Komplexität schwerlich absolut erfasst werden kann [...], resultiert damit aus deren doppelter Relationalität, da letztlich immer zu fragen ist; Komplex im Vergleich und in Bezug zu was?« (Zeman 2017, S. 56)

\section{Einfachheit aus literaturwissenschaftlicher Perspektive}

In der Literaturwissenschaft sind bislang, soweit wir sehen, abgesehen von einzelnen, historisch orientierten Studien zur Simplizität, weder Einfachheit noch Komplexität zum Gegenstand grundlegender Forschung geworden. ${ }^{2}$ Dies hängt vermutlich damit zusammen, dass >Einfachheit< nach weitgehender Übereinstimmung zumindest

\footnotetext{
2 Siehe neben den Hinweisen zur literaturtheoretischen Diskussion bei O'Sullivan (2016) jetzt jüngst die Monographie von Nadine Wisotzki (2021), die für den vorliegenden Beitrag nicht mehr berücksichtigt werden konnte.
} 
in der abendländischen Tradition gerade keine ästhetische Qualität ist. Komplexität dagegen kann als so etwas wie eine Universalie des ästhetischen Diskurses bezeichnet werden, die in verschiedensten Begriffen und Konzepten wie Fülle, Mannigfaltigkeit, Fruchtbarkeit, Reichtum, Vieldeutigkeit oder, jüngeren Datums, Ambiguität zum Ausdruck kommt - aber sich gerade darum ebenfalls kaum als Gegenstand der Forschung anbietet. So sind außer Andrè Jolles klassisch gewordener Studie »Einfache Formen« (1930), die, wie der Titel schon andeutet, eine formengeschichtliche Untersuchung >kleiner < Erzählgattungen ist, erst in jüngerer Zeit Sammelbände vorgelegt worden, die das Thema von »Komplexität und Einfachheit « (Koschorke 2017; vgl. Berndt/Koepnick 2018; Klaiber/Scheiding/Stievermann 2019) zu erschließen beginnen, und die dabei systematisch und im engeren Blick auf die literarische Ästhetik noch ausbaufähig erscheinen. Koschorke (2017) kommt nach einem Abriss der kurzen Begriffsgeschichte der >Komplexität< und der Feststellung, »dass der Begriff der Komplexität im Bereich ästhetischer Phänomene bisher keine allgemein akzeptierte definitorische Schärfung erfahren hat « (Koschorke 2017, S. 6), zu folgendem vorläufigem Definitionsversuch:

Das Maß für ästhetische Komplexität wäre dann die Verwebungsdichte möglicher oder aktualisierter Relationen zwischen den Elementen, die das Kunstwerk umfasst. Der Rechenzeit der maschinellen Algorithmen könnte man dementsprechend die >Deutungszeit< menschlicher Kunstrezipienten zur Seite stellen. Solche Analogiebildungen sind jedoch aus mindestens zwei Gründen problematisch. Erstens würde man sich dadurch auf ein autonomieästhetisches Verständnis geschlossener Kunstwerke festlegen. Zweitens würden sich nicht einmal unter dieser Prämisse die Elemente und damit die möglichen Relata in einem Kunstwerk wie ein feststehendes Set benennen und abzählen lassen. Welche Potentialitäten und Referenzen ein Kunstwerk enthält, ist nicht ein für alle Male quantifizierbar. Es hängt von den sich ständig wandelnden Rahmenbedingungen eines prinzipiell unabschließbaren Prozesses der Rezeption ab. (Koschorke 2017, S. 6)

Die »Verwebungsdichte möglicher oder aktualisierter Relationen zwischen den Elementen « als »Maß für ästhetische Komplexität« entspricht weitgehend dem oben (4.) hergeleiteten quantitätsbezogenen linguistischen Komplexitätsbegriff. Der anschließend als Vorbehalt ihm gegenüber formulierte Hinweis auf den »prinzipiell unabschließbaren Prozess[] der Rezeption«, der sich aus den potentiellen Sinnangeboten und Referenzen eines Kunstwerks wie aus den »sich ständig wandelnden Rahmenbedingungen « seiner aktuellen Rezeption begründet, ist in der linguistischen Diskussion durch das oben referierte Konzept der relativen Komplexität bereits aufgenommen worden. Literarische Komplexität kann demnach einerseits als Textmerkmal erforscht und beschrieben werden, realisiert sich aber andererseits nicht/nie unabhängig von ihrer individuellen Wahrnehmung in der Lektüre - die die textuell angelegte Komplexität wahrnimmt und dabei mindern, aber auch steigern kann. Grundsätzlich gilt dies, unabhängig von der adressierten Lesergruppe, für jeden Rezeptionsakt.

Einfachheit erscheint in Koschorkes Überlegungen nicht als eigenes ästhetisches Konzept (was die oben behauptete Dominanz des Komplexitäts-Paradigmas im äs- 
thetischen Diskurs indirekt bestätigt), sondern nur als Eigenheit des ästhetischen Artefakts (bzw. ästhetischer Produktion) im Verhältnis zu seiner Umwelt, insofern »Ästhetische Formen selektiv [sind], weil sie nur bestimmte Außenweltgehalte in das Kunstwerk einlassen « (Koschorke 2017, S. 7). ${ }^{3}$ Diese > Vereinfachung< tangiert jedoch nicht die »immense Eigenkomplexität« (Koschorke 2017, S. 7), die Kunstwerke dessen ungeachtet entwickeln können. Wo tatsächlich einfach strukturierte Werke vorliegen (im oben erläuterten Sinne >absoluter $<$ Simplizität) - wie z.B. im modernen Minimalismus von Marcel Duchamps Ready-made Fountain oder in Gedichten Ernst Jandls - gehen diese, so Niklaus Largier in demselben Band, doch von »Praktiken« aus, »die Lebenswelten und Reflexionsmuster komplizieren « und erwarten eine Auseinandersetzung, die sie »in der Lektüre und der literaturwissenschaftlichen Interpretation wieder im Blick auf ihre Komplexität befragt. « (Largier 2017, S. 12; mit Fischer und Zeman gesprochen, können also auch strukturell einfache literarische Texte ein hohes Maß an »Benutzerkomplexität« und »Verarbeitungsaufwand « erzeugen bzw. stimulieren, s.o. 4.) Auch aus literaturwissenschaftlicher Perspektive führt der Weg zur Einfachheit demnach nur über die Komplexität. Und auch diese könnte sich quantitativ als eine Menge >möglicher oder aktualisierter Relationen zwischen Elementen< beschreiben lassen - wobei genauer zu klären bzw. zu konkretisieren wäre, was hierbei als >Element< identifiziert werden soll. Zugleich ist, wie deutlich geworden sein sollte, gerade auch im ästhetischen Modus die Relationalität der Komplexität hervorzuheben: Ein sehr einfaches Objekt (wie ein Urinal oder ein Gedicht aus wenigen Nomen) kann Anlass einer sehr komplexen ästhetischen Erfahrung und Reflexion werden (ob und wie eine solche Menge sinnlicher und gedanklicher Operationen wiederum nach den Maßstäben einer >angemessenen oder >professionellen< literarischen Textlektüre einzuschränken ist, wäre ein eigenes Problem).

Insofern aber nun Komplexität ein zwingendes Kriterium des Ästhetischen zu sein scheint (die Gründe für eine solche Auffassung, die die abendländische Modellierung des Ästhetischen überhaupt berühren, sind an dieser Stelle nicht weiter zu erörtern), stellt sich die eingangs aufgeworfene Frage noch einmal neu, ob in Texten vereinfachter Sprache eine ästhetische Dimension erreichbar ist. Auch für diese Frage halten wir eine Skalierung wie auch einen relationalen Komplexitätsbegriff für die geeignete Lösung. Statt einer Entweder-Oder-Entscheidung über das Vorliegen ästhetischer Komplexität wären demnach nach Textmerkmalen der Literarizität (s.o.) und innertextuell verknüpfbaren Elementen zu fragen, die rein informationelle Textorganisation und Textgebrauch durch ein »Mehr an« Textmerkmalen übersteigen und zu einer über die Informationsentnahme hinausgehende (und damit ästhetische) Rezeption eines Textes anregen.

Das Repertoire literarästhetischer Textmerkmale lässt sich nach dem bisher Gesagten in einer offenen und zu erweiternden Liste so zusammenstellen:

\footnotetext{
3 Vgl. demgegenüber die Annahme einer progressiven Zunahme an Komplexität im Kunstwerk von der dissimilativen Versrede zur assimilativen Kunstprosa in der Annäherung an die Komplexität der natürlichen Sprache und nicht-literarischen Welt bei Jurij Lotman, dazu Lypp (1984), S. 25-27, O'Sullivan (2016), S. 20-23.
} 
- Freigestellte Referenz

- Bruch mit konventionell Vorausgesetztem

- (Markierte) Unbestimmtheit

- Lautgestalt

- Sprachspiel

- Unkonventionelle Wortfügungen und Wortstellungen

- Perspektivität der Darstellung

- Verstöße gegen Logik und lineare Chronologie in der Darstellung, akzeptierte Inkonsistenz und Unwahrscheinlichkeit

- Mehrdeutigkeit und Metaphorizität

- Literarische Form- und Gattungssignale

- Reflexivität und Selbstbezüglichkeit

Keines dieser Merkmale ist durch vereinfachten Sprachgebrauch prinzipiell ausgeschlossen, sondern es realisiert sich in mehr oder weniger ausgeprägter, in >einfacherer oder >komplexerer Weise im literarischen Text und unter je individuellen Voraussetzungen in seiner Wahrnehmung durch die Rezipienten. Dieses genauer zu untersuchen und die weitere Entwicklung einer Literatur in vereinfachter Sprache daraufhin zu beobachten, wäre eine Aufgabe künftiger linguistisch-literaturwissenschaftlicher Forschung. Erste Anhaltspunkte finden sich in den vorliegenden Regelwerken und Empfehlungen zum Verfassen von Literatur in Leichter Sprache, die wir im Folgenden vorstellen und abschließend noch durch einige eigene textanalytische Beobachtungen an konkreten Beispielen ergänzen wollen (7.).

\section{Von der Leichten Sprache zur Literatur in Leichter Sprache - Regelwerke, Empfehlungen, Selbstverpflichtungen}

Die Regelwerke zur Leichten Sprache (siehe oben 3.), deren Fokus auf die barrierefreie Vermittlung von Informationen gerichtet ist, enthalten keine Empfehlungen zum Verfassen bzw. Übersetzen von Literatur in vereinfachter Sprache und bestätigen damit indirekt Weimars Literaturdefinition, dass diese jenseits der reinen Informationsentnahme angesiedelt ist (vgl. oben 2.). Vielmehr scheinen Regeln, wie für >gleiche Dinge< > gleiche Wörter < zu verwenden oder gar >bildliche Sprache zu vermeiden<, (vgl. Bredel/Maßß 2016, S. $469 \mathrm{ff}$.), wie oben bereits theoretisch diskutiert, einer Literatur in Leichter Sprache entgegenzustehen. Die Praxis des Handlungssystems Literatur hat jedoch mittlerweile wie schon angesprochen diese Bedenken faktisch überholt.

Bereits 1999 hatte Bror I. Tronbacke in seinen für die International Federation of Library Associations and Institutions (IFLA) zusammengestellten »Guidelines for Easy-to-Read Materials« einen »Bedarf an allen Arten von Easy-Reader Literatur und Informationen « konstatiert und bemerkenswerterweise an erster Stelle »Belletristik und Sachliteratur « in allen Genres gefordert (IFLA1999: 2.1; vgl. Köster 2018, S. 58 ff.). Zugleich konstatierte der Verfasser, dass Versuche, »die Elemente zu identifizieren, die einen leicht lesbaren Text ausmachen «, gescheitert seien (die Neuauflage der Richtlinien 2010 hält an diesem Befund fest) und man stattdessen 
»heute eher auf Erfahrungen « baue (IFLA1999: 3.1). Tronbacke verbindet diese kritische Diagnose mit einem Appell an die »künstlerische Freiheit«:

Die künstlerische Freiheit eines Autoren sollte nicht zu sehr eingeschränkt werden, und dies gilt auch für Schriftsteller, Illustratoren und Fotografen. Diese Erfahrung ist von Bedeutung - es sollte nicht übersehen werden, daß Schreiben und Illustrieren schöpferische Prozesse sind, die, wenn sie zu vielen Einschränkungen unterworfen sind, selten erfolgreich sein können. Daher ist es auch nicht möglich, ein Handbuch für diese Prozesse zu entwerfen.

Man sollte auch nicht zu dogmatisch sein. Belletristik muß Erzählliteratur bleiben. Die Herstellung von Easy-Reader Material sollte den Hersteller anregen und ihm Freude bereiten. Bücher werden von Menschen für Menschen geschaffen, und sollten unterhalten und die Vorstellungskraft anregen. Schriftsteller und andere Künstler sollten die Herausforderung, die die Herstellung von Easy-Reader Material darstellt, erfahren können. (IFLA 1999, Kap. 3.1)

Bezog sich dieser Ratschlag, der Easy-Reader-Programmatik folgend, auf vereinfachende Übersetzungen von Literatur, hat sich die Auseinandersetzung inzwischen auch auf Originalliteratur in Leichter und Einfacher Sprache ausgeweitet und wird nun auch von den Autoren selbst mitgeführt. Das Thema Leichter Sprache hat damit die Poetik erreicht, innerhalb derer seit Aristoteles Ratschläge und Regeln, letztere im Zuge sogenannter präskriptiver >Regelpoetiken $<$, für das Verfassen und Beurteilen von Literatur formuliert worden sind, die in der Moderne schließlich auch experimentellen Charakter angenommen haben (so etwa innerhalb der 1960 gegründeten französischen Gruppe Oulipo). Neu ist allerdings, dass sich poetische Regeln nicht am guten Geschmack, an Schönheit, Spiel oder Wirksamkeit ausrichten, sondern mit Blick auf eine besondere Adressatengruppe eine Restriktion artifizieller Möglichkeiten vorgeben. Die Autorin Alexandra Lüthen hebt letztere als kreative Herausforderung hervor, die

Stellen im Regelwerk [zu finden], an denen sich mir eine Lücke anbietet, die der Text mit seinem Wunsch nach literarischer Flexibilität beantwortet. Mein Arbeiten findet also im Spannungsfeld zwischen Regeln und freiem Wachstum statt. (Lüthen 2019, S. 11)

Auf der anderen Seite konstatiert Lüthen für die Seite der Leser als Erfahrung aus der Praxis die Fähigkeit, durch Kontext und Lesewille angeregt, mehr verstehen zu können, als die Regel vorsieht:

Durch die Einbettung in den Kontext wird auf einmal der Konjunktiv mit verstanden oder ein selten verwendetes Wort erschließt sich aus dem Zusammenhang. Die Leselänge ist nicht mehr so wichtig, weil der Text mitzieht, weil man mehr will, wo man schon mal am Lesen ist. (Lüthen 2019, S. 21)

Als jüngeres Beispiel für die Selbstpositionierung von Autoren im literarischen Feld Einfacher Sprache ist auch die oben bereits angesprochene, aus einer mehrjährigen Lesereihe im Literaturhaus Frankfurt am Main hervorgegangene Anthologie » LiES « zu nennen. Bestandteil des Nachworts des Herausgebers ist eine als Grundlage eines »Experiment[s] « (Hückstädt 2020, S. 260) ausgewiesene Liste mit zehn 
Regeln, »die sich die Autorinnen und Autoren gegeben haben « (Hückstädt 2020, S. 261). Bereits die erste Ankündigung eines 2016 begonnenen Vorläufer-Projekts »Frankfurt, deine Geschichte« stellte diesen Akt autonomer Regelsetzung durch die Autoren selbst heraus (vgl. Pressemitteilung 2016). Die Literarizität ihrer angekündigten Texte brachten die Autoren selbst mit der »Schönheit der Sprache« (Nora Bossong) und der Kraft der autoreigenen »Imagination« (Mirko Bonné) in Verbindung (Pressemitteilung 2016; zitiert auch bei Köster 2018, S. 60) - zwei Aspekten, die in den oben referierten Stellungnahmen zur Möglichkeit von Literatur in Einfacher Sprache interessanterweise keine Rolle spielen (bei Bredel/Maaß 2016, S. 51 wird die widersprechende »negative Zuschreibung[...]«, dass Leichte Sprache »die Schönheit und Komplexität der Sprache nicht erfassen« könne, referiert, aber nicht weiter kommentiert).

Rund die Hälfte der zehn der Anthologie »LiES« zugrunde liegenden Regeln deckt sich mit den auf Informationstexte konzentrierten Leichte-Sprache-Regelwerken (Verwendung einfacher Wörter und einfacher Sätze, möglichst vieler Verben und weniger Nomen, anschauliche Textgliederung, begrenzter Textumfang), während die andere Hälfte der Regeln auf die Spezifik literarischer Texte ausgerichtet ist. Gleich die erste Regel: »In den Texten können wir erfinden« (Hückstädt 2020, S. 261), rekurriert auf die Literarizität (und bestätigt die Dominanz des Fiktionalitätskriteriums als Merkmal von >Literatur $<$ ). Die weiteren literarischen Vorgaben betreffen die Narration (Vermeidung von Zeitsprüngen und Beschränkung auf eine einzige Erzählperspektive) und die Verwendung von Metaphern: »Wenn wir Sprachbilder verwenden, erläutern wir diese« (Hückstädt 2020, S. 261). Die Hervorhebung der Metapher unter den rhetorischen Stilfiguren erklärt sich aus dem Umstand, dass Metaphern »eine kognitiv anspruchsvolle Versprachlichungsstrategie« darstellen (Bredel/Maaß 2016, S. 469), die darum nach den Regelwerken zur Leichten Sprache zu vermeiden bzw. auf das Notwendigste zu reduzieren sind (Bredel/Maaß 2016, S. 473-479). Die Selbstverpflichtung der Autoren, die verwendeten Sprachbilder zu »erläutern«, führt vor diesem Hintergrund allerdings in ein Dilemma: Denn eine Metapher in einem selbstverfassten Text ist offenkundig >notwendig $<$, wenn sie aber >erläutert < werden kann, ist sie im literaturwissenschaftlichen Sinn keine Metapher mehr. Denn Metaphern sind gerade dadurch charakterisiert, dass ihr Bedeutungsgehalt »unscharf oder offen im Rahmen des Bedeutungspotentials der beteiligten Sprachzeichen bleibt « (Zymner ${ }^{3} 2007$, S. 494). So ist es vielleicht kein Zufall, dass gerade diese Regel in der Anthologie »LiES« keine konsequente Anwendung findet. Jens Rühling beispielsweise baut seine Geschichte »Schwere See, einfache Sprache. Meine Reise um das Schwarze Meer« (Hückstädt 2020, S. 101-116) in sehr interessanter Weise um die Frage auf, warum das Schwarze Meer >schwarzes Meer heißt (was keine Metapher ist), die titelgebende Metapher >Schwere See< dagegen bleibt tatsächlich unerläutert. Damit ist bereits angedeutet, dass der Weg von der Reflexion und der Regel zum literarischen Text - zum Glück, möchte man sagen kein linearer ist. Mit dem folgenden Abschnitt richtet sich darum unser Blick zuletzt auf die Literatur selbst. 


\section{Literarische Beispiele: Herrndorf, Lüthen, Schoch}

Haben wir bislang unsere Frage, ob Einfachheit überhaupt mit Literarästhetik vereinbar ist, theoretisch in linguistischer und literaturwissenschaftlicher Hinsicht verfolgt (und - soweit dies aus rein theoretischer Perspektive möglich ist - positiv beantwortet), möchten wir abschließend unsere Überlegungen an drei literarischen Beispielen konkretisieren, die alle ausdrücklich in >einfacher Sprache< verfasst sind. Die im vorliegenden Rahmen kursorisch bleibende Diskussion von literarischen Texten in vereinfachter Sprache widmet sich zunächst einer Übersetzung eines literarischen Originals in Einfache Sprache (Wolfgang Herrndorf, Tschick) und im Anschluss daran zwei Erzählungen, die original in Einfacher Sprache verfasst sind (Alexandra Lüthen, Herzliebe, sowie Julia Schoch, Ich verlasse dich). Übersetzung und Original unterscheiden sich prinzipiell darin, dass bei letzteren die Autorinnen die Gelegenheit nutzen können, Einfachheit im Sinne der Regelwerke vereinfachter Sprache von Vornherein zum Teil ihres literarästhetischen Programms zu machen. Eine vereinfachende Übersetzung hingegen muss sich einerseits immer mit dem Original messen lassen, welches andererseits die Möglichkeiten des kreativen Ausschöpfens der Potenziale von Einfachheit einschränkt. Während die Analyse der beiden Erzählungen in Einfacher Sprache also insbesondere geeignet erscheinen, um Fragen der Ästhetik des Einfachen zu diskutieren, bietet die Übersetzungsanalyse durch den unmittelbaren Vergleich von Übersetzung und Original Anschauungsmaterial für die Diskussion möglicher Formen ästhetischer Komplexität, bieten doch Übersetzung und Original ein ideales empirisches Material für die Kontrastierung von Mehr und Weniger.

\subsection{Wolfgang Herrndorf: Tschick: Original und Übersetzung}

Wolfgang Herrndorfs zuerst 2010 erschienener Roman Tschick um zwei jugendliche Außenseiter ist mittlerweile ein auch durch den Schulunterricht kanonisierter Klassiker der Jugend- und >All-age<-Literatur. Das ist sicherlich auch der Grund dafür, warum mit der Studie von Topalovic/Diederichs (2020) bereits ein in didaktische Diskussionen zum literarischen Lernen eingebetteter Vergleich von Textausschnitten des Romans in Original und Einfache-Sprache-Fassung vorliegt. Für die hier im Fokus stehende Frage nach der Vereinbarkeit von Einfachheit mit Literarästhetik ist besonders interessant, dass Herrndorfs Roman sich nicht nur aus inhaltlichen Gründen für eine Übersetzung anbieten mag, sondern auch über weite Passagen selbst schon durch sprachliche Einfachheit geprägt ist, sodass auf den ersten Blick gefragt werden könnte, ob hier überhaupt eine zusätzliche Vereinfachung notwendig ist. Dies gilt auch für den von uns im Folgenden behandelten kurzen Dialog. Die höhere Komplexität des Originals - das sei hier zu Beginn gleich vorweggenommen - liegt bei ihm denn auch nicht in Satzlänge oder Satzkomplexität begründet, sondern eher im Einsatz von Ellipsen und der teilweise damit verbundenen größeren 
Deutungsoffenheit, die die erste Annäherung der beiden Hauptfiguren des Romans kennzeichnet. ${ }^{4}$

Bei dem Dialog handelt es sich um das erste längere Gespräch zwischen Maik und Tschick, den beiden Protagonisten des Romans (Ende Kapitel 12 im Original; Kapitel »Maik und Tschick« in der ES-Fassung). In Kapitel 12 des Originals bzw. dem vorhergehenden Kapitel »Tatjana « in der ES-Fassung geht es darum, dass Maik sehnsüchtig darauf hofft, von seiner angebeteten Tatjana zum Geburtstag eingeladen zu werden. Der Übergang zum Dialog ist fließend. Tschick unternimmt hier eine Kontaktaufnahme, indem er Maiks Jacke bewundert, nach Maiks Zeugnis fragt und seine Anerkennung darüber äußert, dass Maik sein Zeugnis noch nicht gelesen hat. Als epischer Dialog ist das Gespräch in den Erzähltext eingebettet, der die wörtliche Rede der Protagonisten im Rückblick des beteiligten Maik ergänzt, aber auch kommentiert und auf diese Weise einen fortgesetzten Perspektivwechsel vornimmt, den auch die ES-Fassung beibehält. Für die weitere Analyse der Dialoggestaltung sei der folgende Abschnitt wiedergegeben (ein längerer eingeschobener Erzählerkommentar wird ausgelassen):

»Geile Jacke«, sagte er. »Ich kauf sie dir ab.«

»Das ist meine Lieblingsjacke«, antwortete ich. »Die

verkauf ich nicht.«

[...]

»Nerv ich dich?«, fragte Tschick. »Dann sag

Bescheid!«

Ja klar, dachte ich. Und dann krieg ich eine in die

Fresse, oder was?

»Bist du sitzen geblieben?«, fragte Tschick weiter.

$» \mathrm{Nein} \ll$, antwortete ich.

»Ich frag nur, weil du so mies aussiehst.«

$\mathrm{Na}$ klar, ich sah traurig aus. Ich war sogar richtig

unglücklich. Ich hatte schließlich keine Einladung

bekommen.

»Und was machst du jetzt?«, fragte Tschick.

»Ich geh nach Hause.«

»Und dann?«

»Weiß nicht.«(Herrndorf/Lindemann 2013, S. 14f. $)^{5}$

\footnotetext{
4 Wir gelangen damit zu einem anderen Ergebnis als Topalovic/Diederichs (2020), die der ES-Variante eine geringere syntaktische Komplexität bescheinigen als dem Original. Die unterschiedlichen Ergebnisse unserer Analysen sowie der Analysen von Topalovic/Diederichs zeigen, welche bedeutende Rolle der Auswahl von Textabschnitten für Analysen dieser Art zukommt, wobei hier offenbar der Grad an Dialogizität entscheidend ist. Gerade vor diesem Hintergrund sind die im Folgenden vorzustellenden Ergebnisse nicht als repräsentativ für die Texte in ihrer Ganzheit zu bewerten (wie Topalovic/Diederichs' Einschätzung, dass ihre »Ergebnisse [...] tendenziell auf die Gesamttexte übertragbar« seien, 2020, S. 104, entsprechend zu relativieren). Darauf kommt es hier aber auch nicht an, geht es doch vielmehr um die exemplarische Diskussion der Frage, inwiefern auch einfache Strukturen literarästhetisches Potential entfalten können.

5 Alle literarischen Texte werden im Folgenden exakt mit Leerzeilen und Zeilenfall nach der jeweils angegebenen Druckfassung wiedergegeben, da die Erscheinung des Druckbildes sowohl Gegenstand der Emp-
} 
Der Dialog - ohne die eingeschobenen Erzählanteile (s.u. sowie Abb. 2) - enthält 13 selbstständige Einheiten (also Sätze, Ellipsen oder interaktive Einheiten) und 47 Wortformen. Die durchschnittliche Länge der selbstständigen Einheiten beträgt folglich 3,6 Wortformen. Das Interessante ist nun, dass die durchschnittliche Länge der selbstständigen Einheiten im hier nicht wiedergegebenen Original mit 3,2 sogar noch darunter liegt. ${ }^{6}$ Aus der Perspektive der Satzlänge hat also schon das Original Einfache-Sprache-Qualitäten. Die Werte beider Textversionen liegen deutlich unter den in Korpusstudien ermittelten Durchschnittswerten für die Satzlänge in Leichte- und Einfache-Sprache-Texten (Leichte-Sprache: 9,36; Einfache Sprache: 11,34 [Lange 2018, S. 79]). Für einen unmittelbaren Vergleichseindruck sei der Anfang des Originals zitiert:

[Am Ausgang] [...] haute jemand auf meine Schulter und sagte: »Übertrieben geile Jacke.« Es war Tschick. Beim Grinsen sah man zwei große Zahnreihen, und die Schlitzaugen waren noch schmaler als sonst. »Kauf ich dir ab. Die Jacke. Bleib mal stehen.«

Ich blieb nicht stehen, aber ich hörte, wie er mir nachlief.

»Lieblingsjacke«, sagte ich. »Unverkäuflich.« (Herrndorf ${ }^{19} 2015$, S. 61)

Der kurze Ausschnitt lässt erkennen, wie die extreme Kürze der selbstständigen Einheiten in Herrndorfs »Tschick « zustande kommt: Vollständige Sätze sind anscheinend nicht erlaubt, die knappen, häufig nur aus einem Wort bestehenden Äußerungen sind offenbar - gerade in der Phase der ersten Annäherung - ein Gebot jugendlicher Coolness. Im Abgleich mit unserer Liste literarästhetischer Textmerkmale (Abschnitt 5) kann hier von einem »Bruch mit konventionell Vorausgesetztem « und »unkonventionellen Wortfügungen und Wortstellungen « gesprochen werden. Die Übersetzung in Einfache Sprache hingegen übersetzt den Ausgangstext in ein schriftsprachlich geleitetes konventionelles Stilideal zurück. Das scheinbare satzgrammatische Weniger (die gegenüber der Einfache-Sprache-Version kürzeren syntaktischen Einheiten) im Original geht mit einem Mehr an Figurenzeichnung, an dialogischer Virtuosität (s.u.) und nicht zuletzt an Offenheit in der Bewertung der Figur einher: Am Ende des Dialogs wird in der Übersetzung mit »Ich fand ihn ganz nett « eine Bewertung Tschicks durch den Erzähler vorweggenommen, während am Ende des Originalkapitels noch nicht absehbar ist, dass es sich um den Beginn einer wunderbaren Freundschaft handelt. Die im Original zunächst unklare Bewertung der Figur ließe sich in gewisser Weise den literarästhetischen Merkmalen der »Mehrdeutigkeit « und der »Unbestimmtheit « zuordnen, aber vielleicht besser noch als eine Urteilsenthaltung beschreiben, die literarische Texte nicht selten charakterisiert. Dass die Übersetzung letztere an dieser Stelle auflöst, folgt, so scheint es, dem Gebot der Implikaturvermeidung, dass für die Übersetzung informationsbestimmter Texte einschlägig ist (Maßß 2015, S. 150f.). Literarästhetisch gesehen, ist die

fehlungen der Regelwerke für Texte in vereinfachter Sprache ist (Bredel/Maaß 2016, Kap. 9) als auch ein traditionelles literarisches Gestaltungsmittel, etwa, aber nicht nur, in der Lyrik.

${ }^{6}$ Topalovic/Diederichs (2020) hingegen ermitteln für die von ihnen untersuchten Textausschnitte: das erste Kapitel der ES-Fassung und die entsprechenden Kapitel 9 und 10 des Originals, eine durchschnittliche Satzlänge von 16,1 Wörtern im Original und 6,6 Wörtern in der ES-Fassung. Das ist zweifelsohne - wie bereits in Fußnote 4 angemerkt - auf die jeweilige Auswahl eines Textausschnitts zurückzuführen. 
konsequente Explikation von Mitgemeintem unmöglich, an vorliegender Stelle und grundsätzlich berührt der Umgang mit ihr die Frage, wieviel Zumutung (Abschnitt 2) ein Text in vereinfachter Sprache enthalten soll.

Wenngleich in Original und Übersetzung in der zitierten Passage die gleichen Themen verhandelt werden - Bewertung der Jacke, Zeugnis, weiterer Verlauf des Tages - ist der Originaldialog mit 136 Wortformen und 43 selbstständigen Einheiten fast dreimal so lang wie die ES-Fassung. Das liegt daran, dass der Dialog sozusagen mehr Schleifen nimmt. Abbildung 2 verdeutlicht die in dieser Hinsicht höhere Komplexität der Dialogstruktur im Original.

Die Analyse der Gesprächsphasen erfolgt als eine Kombination aus gesprächsanalytischer Dialoganalyse (Spiegel/Spranz-Fogasy 2001) sowie einer Analyse der Verzahnung von dialogischen und erzählenden Passagen im Romanausschnitt. Der Abbildung kann entnommen werden, dass die höhere Textlänge des Originals auch mit einem Mehr an Dialog- und Erzählpassagen einhergeht. Der Unterschied liegt darin, dass die beiden Kernphasen des Dialogs - »Jacke« und »Zeugnis « - in der Übersetzung jeweils als eine kurze Dialogpassage realisiert sind, während sie im Original in jeweils mehrere, mit Erzählerberichten und -kommentaren verzahnten Abschnitte aufgeteilt sind. Dabei wird Kernphase 2 von Kernphase 1 gerahmt.

Aber auch die einzelnen Dialogpassagen des Originals weisen in vielen Fällen ein Mehr an Sprecherwechseln auf. Dass diesen das literarästhetische Textmerkmal Selbstbezüglichkeit und Sprachspiel attestiert werden kann, soll die folgende Dialogpassage illustrieren, in der Tschick einleitend seine Annahme begründet, Maik könnte sitzengeblieben sein:

»Aber lauter Fünfen«, sagte er. »Keine Ahnung.«»Wie, keine Ahnung? Wenn ich dich nerv, mach Meldung.« Ich sollte mich melden, dass er mich nervte? Und dann kriegte ich eins in die Fresse, oder was? »Weiß ich nicht. «»Du weißt nicht, ob ich dich nerv?«»Ob ich lauter Fünfen hab.«»Im Ernst?« »Ich hab noch nicht reingeguckt.«»In dein Zeugnis? « Nein.«»Du hast in dein Zeugnis nicht reingeguckt?«»Nein.«»Echt? Du hast dein Zeugnis gekriegt und nicht reingeguckt? Wie cool ist das denn. « (Herrndorf ${ }^{19} 2015$, S. 62 f.)

Der kurze Ausschnitt enthält sieben Gesprächsbeiträge (Turns) von Tschick und sechs von Maik. Im Gegensatz kommt die Übersetzung mit vier - meist durch Erzählpassagen und nicht durch Wechselrede voneinander abgegrenzten - Äußerungen von Tschick aus, Maik ist ausschließlich mit dem Responsiv »Nein« an der Dialogpassage beteiligt:

»Nerv ich dich?«, fragte Tschick. »Dann sag

Bescheid!«

Ja klar, dachte ich. Und dann krieg ich eine in die

Fresse, oder was?

»Bist du sitzen geblieben?«, fragte Tschick weiter.

$»$ Nein«, antwortete ich.

»Ich frag nur, weil du so mies aussiehst.«(Herrndorf/Lindemann 2013, S. 14 f.)

Ein Mehr an Dialogizität muss noch nicht zwingend ein Mehr an Literarästhetik bedeuten. Der rasante Wechsel an Gesprächsbeiträgen hat aber - neben seiner 
Abb. 2 Dialogphasen und ihre Verzahnung mit der Erzählstruktur in Herrndorfs »Tschick « (Original und Übersetzung)

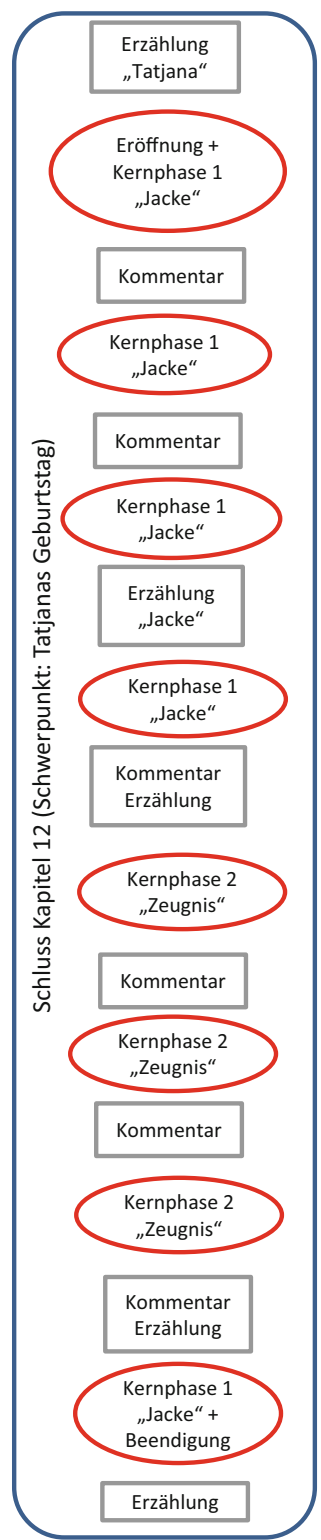

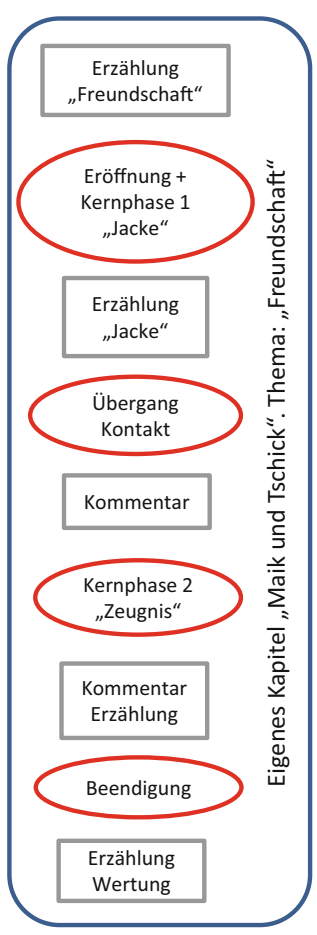

Mündlichkeit stilisierenden Funktion (Hennig/Jacob 2016) - auch sprachspielerische Qualitäten. Diese zeigen sich besonders deutlich im Spiel mit dem doppeldeutigen Bezug von Maiks Äußerung Weiß ich nicht. Dass diese Doppeldeutigkeit im Wortwechsel zwischen den beiden expliziert und schließlich aufgelöst wird: »Weiß ich nicht. « »Du weißt nicht, ob ich dich nerv? « »Ob ich lauter Fünfen hab. « erfüllt interessanterweise auch im Original die Vorgabe der Einfachen Sprache für literarisches wie nicht-literarisches Formulieren, kognitiv anspruchsvollere Sprachformen zu >er- 
läutern<, wenn sie unumgänglich sind. Herrndorf gibt an dieser Stelle ein Beispiel dafür, wie diese >Aufgabe< nicht umständlich und illusionsdurchbrechend an einen extradiegetischen Erzähler delegiert werden muss, sondern auch innerdiegetisch gelöst werden kann.

Als Fazit dieses kurzen Vergleichs können wir festhalten: Einfache Sprache ist nicht auf Literatur in vereinfachter Sprache beschränkt, sondern ein mögliches Textund Stilmerkmal von Literatur überhaupt - als solche kann sie aber, wie im vorliegenden Fall, die Übersetzung in vereinfachte Sprache auch vor eigene Herausforderungen stellen. Die Vereinfachung der Übersetzung findet insgesamt eher auf der Ebene der Dialog- und Erzählstruktur statt als im Bereich der wort- und satzgrammatischen Ausgestaltung. M.a.W: Auch das Original folgt im Wesentlichen dem »Eine Aussage pro Satz«-Prinzip (Bredel/Maaß 2016, S. 105) und enthält keine Genitive, Passive, Präteritumsformen oder längere Komposita und nur einen Konjunktiv. Das Original enthält drei Nebensätze, die Übersetzung einen (aufgrund der unterschiedlichen Länge ist das ein Gleichstand). Auch der Wortschatz ist prinzipiell vergleichbar, andererseits sind die Anleihen bei der Jugendsprachlichkeit im Original ein konstitutives literarästhetisches Merkmal des Textes. Die größere Ellipsenfreudigkeit des Originals passt zu Zemans Zuordnung der Ordnungszustände »Regularität« zu Simplizität und »Irregularität« zu Komplexität (das Beispiel verdeutlicht aber auch, dass Regularitäten mediengebunden sind - hier Mündlichkeit/Schriftlichkeit - und von kulturellen Konventionen abhängen). Die elliptischen Strukturen, die, wie bereits ausgeführt, zum rekordverdächtig niedrigen Durchschnittswert der Länge syntaktischer Einheiten im Tschick-Original führen, sind also paradoxerweise gerade nicht aufgrund ihrer Kürze besonders einfach, sondern können als grammatisches Mittel zur Kennzeichnung von Unbestimmtheit und literarisch evozierter Wirklichkeitsnähe (effet de réell) als literarästhetisches Textmerkmal eingeordnet werden.

Wenn wir nun dieses Ergebnis mit der in Abschnitt 5 erstellten Liste potentieller literarästhetischer Textmerkmale abgleichen, so können wir dem Original Merkmale wie Bruch mit konventionell Vorausgesetztem, unkonventionelle Wortwahl, Unbestimmtheit und Mehrdeutigkeit bzw. Deutungsenthaltung attestieren. In der vorliegenden Übersetzung wird auf diese Mittel weitestgehend verzichtet (vgl. die in dieser Hinsicht übereinstimmende Analyse anhand anderer Passagen von Topalovic/Diederichs 2020, S. $107 \mathrm{f}$.), es wird lediglich die Jugendsprache durch geil angedeutet und die Bruchstückhaftigkeit der Äußerungen ansatzweise übernommen, die Literarizität ist aber auch nicht ganz verschwunden. So zeigt sich auch hier, dass einfach und komplex skalare Größen sind. Wie eng die Grenzen literarischer Rede in vereinfachter Sprache tatsächlich gezogen sind, hängt auch von der eigenen Erfindungskraft der Übersetzung und von dem Maß an Zumutung ab (vgl. Fix 2017), das sie einsetzen will, um zu »Literacy-Erfahrungen« anzuregen (Topalovic/Diederichs 2020, S. 108).

Die Annahme liegt nahe, dass einer Übersetzung eines literarischen Originals in vereinfachte Sprache von Vornherein eingeschränktere Möglichkeiten der Entfaltung literarästhetischer Qualitäten zur Verfügung stehen als einem literarischen Text, der nicht auf einer Vorlage beruht, sondern als Original in vereinfachter Sprache konzipiert ist - auch wird eine Übersetzung zwangsläufig immer am Original gemessen, sodass der Eindruck einer reduzierten literarästhetischen Qualität kaum 
zu vermeiden ist. Wir wenden uns deshalb abschließend zwei Beispielen literarischer Originaltexte in vereinfachter Sprache zu.

\subsection{Alexandra Lüthen: Herzliebe und Julia Schoch: Ich verlasse dich: Erzählen in Einfacher Sprache}

»In der Praxis geschieht dann aber glücklicherweise das, was immer passiert:

Die Leser lesen einfach, was sie wollen! Na so was!« (Lüthen 2019, S. 21)

Alexandra Lüthen ist eine Autorin, die sich in den letzten Jahren stark auf das Verfassen von Texten in vereinfachter Sprache konzentriert hat und bereits mehrfach mit Preisen hierfür ausgezeichnet wurde. In ihrem gerade in Hinblick auf unsere Fragestellung äußerst lesenswerten Plädoyer »Allen eine Chance. Warum wir Leichte Sprache brauchen « (Lüthen 2019) hat sie ihre literarische Arbeit auf diesem Gebiet zudem auch für ein breiteres Publikum reflektiert. Ihre Erzählung »Herzliebe« wurde im Rahmen des vierten Literaturwettbewerbs »Die Kunst der Einfachheit« von Mitgliedern aus mehreren Leseklubs von Menschen mit und ohne Behinderung für eine Veröffentlichung ausgewählt (Lebenshilfe 2018, S. 7). Julia Schoch, ebenfalls mehrfach ausgezeichnete Autorin, verfasste ihre erste Erzählung in vereinfachter Sprache »Ich verlasse dich « für die Reihe »LiES! « des Frankfurter Literaturhauses (vgl. Abschnitt 6). Sie erschien 2020 in der gleichnamigen Anthologie. Wir wählen jeweils den Anfang beider Erzählungen:

Ich bin Feli. Felicitas. Das ist ein alter Name.

Er bedeutet: Glück. Ich bin nicht so alt wie der Name.

Ich bin 21 und habe das Leben noch vor mir. So sagt man.

Ich habe auch schon viel Leben hinter mir. Das kann man lesen. In Arzt-Berichten. Es gibt so viele Berichte über mich.

Die Ordner stehen im Regal neben den Foto-Alben.

Auf den Fotos sieht man mich als Baby. Ganz klein.

Ich bin viel zu früh auf die Welt gekommen. Ich konnte es

wohl nicht abwarten mit meinem Leben anzufangen.

Die ersten Fotos sind von mir in einem Krankenhaus.

In meinem Gesicht klebt ein Schlauch. Das Klebe-Pflaster

sieht aus wie ein Herz auf meiner Wange.

Das haben die Pfleger im Krankenhaus so gemacht.

Weil es das Netteste ist, was man tun kann.

Wenn ein Baby es schwer hat und Schmerzen. Dann klebt man Liebe auf die Haut.

Auf mein Herz konnte man kein Pflaster kleben. Das musste operiert werden. Und in Ruhe heilen, tief in mir drin. Das hat es gemacht.

Aber es ist nie ganz gesund geworden. Das war okay.

Jeder hat irgendwas, das nicht ganz gesund ist. (Lüthen 2018, S. 63) 
$* * *$

Es ist ganz einfach:

Ich verlasse dich.

Morgen gehe ich weg. Weg von dir.

Das bedeutet, ich gehe weg von uns.

Es klingt sehr einfach. Aber ich habe

viele Fragen.

Was passiert mit unserer Geschichte, wenn ich gehe?

Wer wird sich um unsere Geschichte kümmern, wenn es uns als Paar nicht mehr gibt?

Ich finde, unsere Geschichte ist wie ein Kind.

Wir tragen die Verantwortung für sie.

Das hört sich komisch an. Ich weiß.

Ich möchte, dass unsere Geschichte auch

ohne uns weitergeht.

Aber solche Sachen sind nicht möglich.

Außer im Traum.

Ich verlasse dich.

Drei Wörter, die jeder versteht. Eigenartig.

Es reichen drei Wörter, und alles ist getan.

Man muss sie bloß aussprechen.

Ich bin erstaunt, dass es so einfach ist.

Bevor ich den Satz laut ausspreche, übe ich ihn im Stillen.

Ich sage ihn vor mich hin.

Ich. Verlasse. Dich.

Jetzt bin ich noch mal erstaunt.

Der Satz ist genauso kurz wie der Satz, den ich am Anfang unserer Geschichte gesagt habe.

Am Anfang habe ich zu dir gesagt:

Ich liebe dich. (Schoch 2020, S. 139)

Beide Textausschnitte belegen, dass die Autorinnen mit den Regeln und Empfehlungen für vereinfachte Sprache sehr gut vertraut sind und gleichzeitig genügend künstlerisches Selbstvertrauen an den Tag legen, um die Regeln nicht zu einem Korsett werden zu lassen. So verzichten beiden Autorinnen bspw. nicht vollständig auf Nebensätze im Sinne der »Eine Aussage pro Satz«-Regel (Relativsätze, Kausalsätze, Objektsätze). Lüthen nutzt auch die Leichte-Sprache-Konvention, einen Nebensatz als eigenständigen orthographischen Satz zu realisieren (Weil es das Netteste ist..., Wenn ein Baby es schwer hat...). Da durch die einfachen Satzgefüge aber noch keine Satzkomplexität entsteht, wird der Eindruck von Einfachheit dadurch nicht geschmälert. Beide Texte enthalten vielmehr sehr kurze syntaktische Einheiten: Auf 
190 Wortformen kommen in »Herzliebe « 30 mit einem Punkt abgegrenzte Einheiten (Durchschnitt: 6,33), in »Ich verlasse dich « sind es 31 auf 174 Wortformen (Durchschnitt: 6,61). Der Einfachheit halber wurde dieser Auswertung ein orthographischer Satzbegriff zugrunde gelegt. Das scheint auch deshalb gerechtfertigt, weil die Auslagerung potentiell integrierbarer Teile in eine neue Einheit (etwa Lüthen: Das kann man lesen. In Arzt-Berichten.; Aber solche Sachen sind nicht möglich. Außer im Traum.; Schoch: Morgen gehe ich weg. Weg von dir.) ganz offenkundig Teil des ästhetischen Programms ist. Mit der künstlich verkürzten Einheitenbildung entsprechen die Autorinnen jedoch nicht nur regelhaft den Anforderungen an vereinfachte Sprache, sondern sie refunktionalisieren das Einfachheitsgebot poetisch. Zunächst öffnet die extreme Segmentierung dem mündlichen Vortrag (vgl. Abschnitt 2) eine hohe Spannbreite an Gestaltungs- und Deutungsmöglichkeiten. ${ }^{7}$ Eine Sinngebung eigener Art vollzieht die Transformation von Ich verlasse dich in Ich. Verlasse. Dich. am Ende des zweiten Absatzes von Schochs Erzählung, die in der asyndetischen Reihe neben der gesteigerten Nachdrücklichkeit der Lossagung auch die Verklammerung von Ich und Dich wahrnehmbar macht, die in der Alltagsrede unauffällig bleibt (im russischen Formalismus ist dieser von dem Konventionsbruch ausgehende Effekt als >Desautomatisierung < beschrieben und zum Grundprinzip des Ästhetischen erhoben worden, vgl. Sklovskij 1969 [1916]). Die ganze Eingangspassage lässt sich in diesem Sinne als künstliche Komplexitätssteigerung einer einfachsten Aussage lesen, als eine poetische Entfaltung von Reflexivität und sprachlicher Selbstbezüglichkeit, die man als solche verstehen kann, aber nicht verstehen muss, um dem Text dennoch folgen zu können. Am Ende der zitierten Passage lenkt die Analogie Ich verlasse dich / Ich liebe dich - zum Kern der Erzählung zurück, in der es darum geht, dass in einem solchen Dreiwortsatz ein ganzes Leben stecken kann.

Eine besondere Bedeutung als literarästhetische Merkmale und Mittel der Komplexitätssteigerung kommen schließlich auch der Erzählperspektive und der Metaphorizität zu. Nicht ohne Grund werden gerade diese beiden Gestaltungsmittel ausdrücklich in der >Selbstverpflichtung < der Frankfurter LiES!-Regeln angesprochen. Statt eine verwendete Metapher eigens zu erläutern, wie die LiES!-Regeln es empfehlen, ${ }^{8}$ kann man an Lüthens Eingangsabschnitt jedoch beobachten, wie einfallsreich eine Metapher ein- und gewissermaßen auch wieder ausgeführt werden kann, ohne sie eigens zu explizieren. So wird der metaphorische Ausdruck Dann klebt man Liebe auf die Haut in der Mitte des dritten Absatzes über mehrere Zeilen vorbereitet (In meinem Gesicht ...), in denen die Sachbereiche >Haut<, >Kleben $<$, >Zuwendung < zunächst einzeln etabliert werden, bevor sie in Dann klebt man Liebe auf die Haut metaphorisch schließlich zur Deckung kommen, um unmittelbar im Anschluss die tröstende Geste mit der nüchternen Realität der lebensbedrohlichen Krankheit zu konfrontieren: Auf mein Herz konnte man kein Pflaster kleben.

Die Beschränkung der literarischen Darstellung auf eine einzige Erzählperspektive halten beide Autorinnen mit der durchgehend verwendeten Ich-Form ein. Beide Texte zeigen jedoch auch, dass eine Perspektivenvielfalt der Darstellung damit keineswegs ausgeschlossen ist. In Lüthens Erzählung sind es gleich zu Beginn die

\footnotetext{
7 Wir hatten die Gelegenheit, beide Autorinnen beim Vortrag ihrer Texte dabei zu erleben.

8 Siehe oben Abschnitt 6, S. \%\%.
} 
Arzt-Berichte, die unausgesprochen eine eigene Perspektive präsentieren, im weiteren Verlauf kommen vor allem das kranke Herz der Protagonistin und die fremde Herzspenderin hinzu, die jeweils eine eigene Gestalt gewinnen. Schochs Text dagegen weist zwar eine äußerlich weitgehend monologische Struktur auf, die aber als Liebes- und Trennungsgeschichte inhaltlich essentiell an eine zweite Perspektive gebunden bleibt, die am Ende der Geschichte in überraschender Weise explizit wird und in einem kurzen Dialog zwischen der Erzählerin und ihrem ahnungslosen Ehemann endet, in dem sich die Trennungsabsicht in ein ungewolltes (und verständnislos aufgenommenes) Liebesgeständnis verkehrt.

Eine stärkere Herausforderung für die Diegese ist, wie sich beobachten lässt, der Verzicht auf das Präteritum, wie sie die Leichte-Sprache-Regelwerke vorsehen (Bredel/Maaß 2016, S. 113, 443 ff.). Sparen die Frankfurter LiES!-Regeln diesen Aspekt aus, realisieren Lüthen und Schoch weitgehend die Vorgabe und zeigen, wie phantasievoll man mit ihr umgehen kann. Denn für beide Erzählungen ist die erzählende Aufarbeitung der erlebten Vergangenheit, die zur gegenwärtigen Situation geführt hat, unerlässlich. So finden sich in beiden Texten - gegen die Empfehlung wiederholt Zeitsprünge, die jedoch interessante Strategien aufweisen, Gegenwart und Vergangenheit nachvollziehbar motiviert gegenüberzustellen. So weisen im Eingangsabschnitt von Herzliebe die neben den Arztberichten platzierten Fotoalben als Dokumentation der Vergangenheit den Einstieg in die Geschichte des kranken Herzens. Wogegen Schoch zu Beginn in eine virtuose Präsenserzählung Vergangenheit, Gegenwart und Zukunft von Liebe und Verlust, ihr Anfang und Ende, die Erinnerung, das Vergessen und schließlich die Zeitenthobenheit im Traum integriert und ansonsten nur vereinzelt auf das Perfekt als eindeutig Vergangenheit markierendes Tempus ausweicht (Am Anfang habe ich zu dir gesagt). Einzig die futurische Formulierung Wer wird sich um unsere Geschichte kümmern weicht deutlich ab - und interessanterweise wäre sie leicht zu vermeiden gewesen (>Wer kümmert sich um unsere Geschichte? $\prec$, was ein indirekter Hinweis auf die besondere Bedeutung der gewählten Formulierung ist. Wer wird sich um unsere Geschichte kümmern, wenn es uns als Paar nicht mehr gibt?, ist die selbstbezügliche Frage, deren unausgesprochene Antwort das Erzählen legitimiert: Es sind die Autorin und ihre Leser, die sich um diese Geschichte kümmern.

Einfache und Leichte Sprache schließt die Literatur nicht aus. Genuin literarästhetische Merkmale lassen sich auch in Originalliteratur und Übersetzungen in vereinfachter Sprache identifizieren, wie unsere Beispiele gezeigt haben. Dass die Grenzen in ihnen enger gezogen sind als in weitgehend regelbefreiter Literatur, ist nicht zu bestreiten - ebenso wenig wie die Plausibilität, Komplexität (Reichtum, Fülle) als ein Merkmal des Ästhetischen und seiner Wahrnehmung einzusetzen. Aber wir hoffen gezeigt zu haben, dass die Grenzen der Darstellungsleistungen vereinfachter Literatur beträchtlich weiter gesteckt sind, als es zunächst den Anschein haben mag, und dass vor allem skalierte Übergänge zwischen dem Einfachen und dem Komplexen in der Theorie wie in der literarischen Praxis zu beobachten und zu analysieren sind. 
Funding Open Access funding enabled and organized by Projekt DEAL.

Open Access Dieser Artikel wird unter der Creative Commons Namensnennung 4.0 International Lizenz veröffentlicht, welche die Nutzung, Vervielfältigung, Bearbeitung, Verbreitung und Wiedergabe in jeglichem Medium und Format erlaubt, sofern Sie den/die ursprünglichen Autor(en) und die Quelle ordnungsgemäß nennen, einen Link zur Creative Commons Lizenz beifügen und angeben, ob Änderungen vorgenommen wurden.

Die in diesem Artikel enthaltenen Bilder und sonstiges Drittmaterial unterliegen ebenfalls der genannten Creative Commons Lizenz, sofern sich aus der Abbildungslegende nichts anderes ergibt. Sofern das betreffende Material nicht unter der genannten Creative Commons Lizenz steht und die betreffende Handlung nicht nach gesetzlichen Vorschriften erlaubt ist, ist für die oben aufgeführten Weiterverwendungen des Materials die Einwilligung des jeweiligen Rechteinhabers einzuholen.

Weitere Details zur Lizenz entnehmen Sie bitte der Lizenzinformation auf http://creativecommons.org/ licenses/by/4.0/deed.de.

\section{Literatur}

Adamzik, Kirsten (2004): Textlinguistik. Eine einführende Darstellung. Tübingen: Niemeyer.

Antos, Gerd (2017): »Leichte Sprache als Politolekt - Anmerkungen zu den Einflussfaktoren: Verständlichkeit, Fremdheit und Transaktionskosten«. In: Bock/Lange/Fix (2017), S. 129-144.

Baumert, Andreas (2016): Leichte Sprache - Einfache Sprache. Literaturrecherche - Interpretation - Entwicklung. Hannover: Bibliothek der Hochschule Hannover.

Berndt, Frauke/Koepnick, Lutz (Hg.) (2018): Ambiguity in contemporary art and theory. Hamburg: Meiner (Sonderheft der Zeitschrift für Ästhetik und allgemeine Kunstwissenschaft 16).

Bock, Bettina M. (2015): »Zur Angemessenheit Leichter Sprache. Aus Sicht der Linguistik und aus Sicht der Praxis «. In: Aptum 11, S. 131-140.

Bock, Bettina M. (2017): »Das Passiv- und Negationsverbot >Leichter Spracheく auf dem Prüfstand. Empirische Ergebnisse aus Verstehenstest und Korpusuntersuchung«. In: Sprachreport 33, S. 20-28.

Bock, Bettina M. (2018): »Leichte Sprache« - Kein Regelwerk. Sprachwissenschaftliche Ergebnisse und Praxisempfehlungen aus dem LeiSA-Projekt. Leipzig. In: https://nbn-resolving.org/urn:nbn:de:bsz: 15-qucosa2-319592 (16.02.2022) [Korrigierte Druckfassung: Wiesbaden: Frank \& Timme, 2019 (Kommunikation - Partizipation - Inklusion 5)].

Bock, Bettina M./Fix, Ulla (2017): »Im Spannungsfeld zwischen Forschung und Praxis - Überlegungen zum >Leichte Sprache<-Band von Ursula Bredel und Christiane Maß«. In: Zeitschrift für germanistische Linguistik, S. 130-149.

Bock, Bettina M./Lange, Daisy (2017): »Empirische Untersuchungen zu Satz- und Textverstehen bei Menschen mit geistiger Behinderung und funktionalen Analphabeten«. In: Bock/Fix/Lange (Hg.), S. 253-274.

Bock, Bettina M./Fix, Ulla/Lange, Daisy (Hg.) (2017): »Leichte Sprache« im Spiegel theoretischer und angewandter Forschung. Berlin: Frank \& Timme (Kommunikation - Partizipation - Inklusion 1).

Bock, Bettina M./Lange, Daisy/Fix, Ulla (2017): »Das Phänomen >Leichte Sprache< im Spiegel aktueller Forschung - Tendenzen, Fragestellungen und Herangehensweisen«. In: Bock/Fix/Lange (Hg.), S. 11-31.

Bredel, Ursula/Maß, Christine (2016): Leichte Sprache: theoretische Grundlagen, Orientierung für die Praxis. Berlin: Dudenverlag.

Christmann, Ursula/Groeben, Norbert (1996): »Textverstehen/Textverständlichkeit: Ein Forschungsüberblick unter Anwendungsperspektive«. In Hans Krings (Hg.): Wissenschaftliche Grundlagen der technischen Kommunikation. Tübingen: Narr (Forum für Fachsprachen-Forschung 32), S. 129-190.

Christmann, Ursula (2017): »Wie leicht darf Leichte Sprache sein? Empirische Lücken in einem gut gemeinten Konzept«. In: Bock/Fix/Lange (Hg.), S. 35-52.

Dahl, Östen (2004): The Growth and Maintenance of Linguistic Complexity. Amsterdam/Philadelphia: Benjamins.

Fischer, Klaus (2017): »Komplexität - dennoch ein nützlicher Begriff«. In: Hennig (Hg.), S. 19-52. 
Fix, Ulla (2017): »>Schwere< Texte in >Leichter Sprache< - Voraussetzungen, Möglichkeiten und Grenzen (?) aus textlinguistischer Sicht «. In: Bock/Fix/Lange (Hg.), S. 163-188.

Forschungsstelle Leichte Sprache (2020): Erkenntnis und Transfer. Barrierefreie Kommunikation als gesellschaftliche Aufgabe und Gegenstand der Forschung. In: https://www.uni-hildesheim.de/ media/fb3/uebersetzungswissenschaft/Leichte_Sprache_Seite/Publikationen/2020_-_Erkenntnis_ und_Transfer._Broschuere_Forschungsstelle_Leichte_Sprache_201209_RZ.pdf (16.02.2022).

Frickel, Daniela A. (2018): »Textpassung. Theoretische und empirische Ansätze zur Ermittlung der Gegenstandsadäquanz von (literarischen) Texten zwischen >Einfachheit< und >Komplexität««. In: Jan Boelmann (Hg.): Empirische Forschung in der Deutschdidaktik. Bd. 3: Forschungsfelder. Baltmannsweiler: Schneider Verlag Hohengehren, S. 185-201.

Gernet, Katharina (2017): Märchen erzählen in Leichter Sprache. Das Praxisbuch. Mit Methoden, Regeln und 12 ausgearbeiteten Märchen. München: Don Bosco.

Goethe, Johann Wolfgang/Fröhlich, Holger (2019): »Leichte Sprache. Ich tu mir so leid. Deswegen will ich jetzt zaubern lernen [»Faust I«, Szene >Nacht<, Übersetzung in Leichter Sprache]«. In: brand eins Magazin 11, 135. In: https://www.brandeins.de/magazine/brand-eins-wirtschaftsmagazin/2019/ qualitaet/ich-tu-mir-so-leid-deswegen-will-ich-jetzt-zaubern-lernen (16.02.2022).

Hauptmeier, Helmut/Schmidt, Siegfried J. (1985): Einführung in die empirische Literaturwissenschaft. Braunschweig/Wiesbaden: Vieweg.

Hennig, Mathilde (Hg.) (2017): Komplexität - ein Phantom? Tübingen: Stauffenburg.

Hennig, Mathilde/Jacob, Joachim (2016): »Nähe, Distanz und Literatur«. In: Mathilde Hennig/Helmuth Feilke (Hg.): Zur Karriere von >Nähe und Distanz. Rezeption und Diskussion des Koch-Oesterreicher-Modells. Berlin/Boston: de Gruyter (Germanistische Linguistik 306), S. 187-211.

Herrndorf, Wolfgang ( $\left.{ }^{19} 2015\right)$ : Tschick [2010]. Reinbek bei Hamburg: Rowohlt.

Herrndorf, Wolfgang/Lindemann, Andreas (2013): Tschick. In Einfacher Sprache. Münster: Spaß am Lesen.

Hückstädt, Hauke (Hg.) (2020): LiES. Literatur in Einfacher Sprache. Geschichten von Alissa Walser, Anna Kim, Arno Geiger, Henning Ahrens, Jens Mühling, Judith Hermann, Julia Schoch, Kristof Magnusson, Maruan Paschen, Mirko Bonné, Nora Bossong, Olga Grjasnowa und Ulrike Almut Sandig. München: Piper 2020.

IFLA (1999): International Federation of Library Associations and Institutions: Richtlinien für Easy-Reader Material. Hg. und zusammengestellt v. Bror I. Tronbacke, unter den Auspizien des IFLA Bereiches Bibliotheksdienste für benachteiligte Personen. Übers. v. Antje Cockrill (IFLA Professional Reports 57). Den Haag: IFLA Zentrale [überarbeitete Neuauflage 2010 (Professional Reports 120)].

Imo, Wolfgang/Lanwer, Jens Philipp (2017): »Sprache ist komplex. Nur: Für wen?« In: Hennig (Hg.), S. 149-174.

Ingarden, Roman (1997): Vom Erkennen des literarischen Kunstwerks [1968]. Hg. von Rolf Fieguth/ Edward M. Swiderski (Gesammelte Werke 13). Tübingen: Niemeyer.

Jacob, Joachim (2016): »Literatur«. In: Werner Holly/Ludwig Jäger/Peter Krapp/Samuel Weber/Simone Heekeren (Hg.): Sprache - Kultur - Kommunikation. Ein internationales Handbuch zu Linguistik als Kulturwissenschaft/Language - Culture - Communication. An International Handbook of Linguistics as Cultural Study (Handbücher zur Sprach- und Kommunikationswissenschaft 43). Berlin/Boston: de Gruyter, S. 490-496.

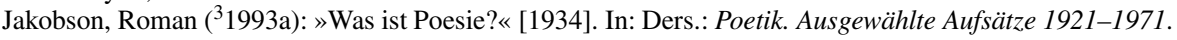
Hg. von Elmar Holenstein/Tarcisius Schelbert. Frankfurt a.M.: Suhrkamp, S. 67-82.

Jakobson, Roman ( $\left.{ }^{3} 1993 b\right)$ : »Linguistik und Poetik« [1960]. In: Ders.: Poetik. Ausgewählte Aufsätze 1921-1971. Hg. von Elmar Holenstein/Tarcisius Schelbert. Frankfurt a.M.: Suhrkamp, S. 88-94.

Jolles, André (1930): Einfache Formen. Legende, Sage, Mythe, Rätsel, Spruch, Kasus, Memorabile, Märchen, Witz. Halle a.d.S.: Niemeyer.

Klaiber, Isabell/Scheiding, Oliver/Stievermann, Jan (Hg.) (2019): Simplify, simplify! Brevity, plainness and their complications in American literature and culture. Festschrift for Bernd Engler on the occasion of his 65th birthday. Paderborn: Ferdinand Schöningh (Beiträge zur englischen und amerikanischen Literatur 37).

Kortmann, Bernd/Szmrecsanyi, Benedikt (Hg.) (2012): Linguistic complexity. Second language acquisition, indigenization, contact. Berlin/Boston: de Gruyter (linguae \& litterae 13), S. 6-34.

Koschorke, Albrecht (Hg.) (2017): Komplexität und Einfachheit. DFG-Symposion 2015. Stuttgart: J.B. Metzler.

Köster, Juliane (2018): »Literatur in einfacher Sprache«. In: Der Deutschunterricht 70, S. 58-67. 
Lange, Daisy (2018): »Comparing >Leichte Sprache<, >einfache Spracheく and >Leicht Lesen<: A CorpusBased Descriptive Approach«. In: Proceedings oft the 1st Swiss Conference on Barrier-free Communikation. Zürcher Hochschule für Angewandte Wissenschaften. Winterthur 2017, S. 75-91.

Lange, Daisy (2019): »Der Genitiv in der >Leichten Sprache<- das Für und Wider aus theoretischer und empirischer Sicht«. In: Zeitschrift für Angewandte Linguistik 70, S. 37-72.

Largier, Niklaus (2017): »Sektion 1: Praktiken der Einfachheit. Einführung«. In: Koschorke (Hg.), S. 11-18.

Lebenshilfe Berlin (Hg.) (2015): Die Kunst der Einfachheit. Geschichten in Einfacher Sprache. Marburg: Lebenshilfe-Verlag.

Lebenshilfe Berlin (Hg.) (2017): Volle Lotte. Liebe muss man fühlen. Marburg: Lebenshilfe-Verlag.

Lebenshilfe Berlin (Hg.) (2018): Gerettet. Geschichten in einfacher Sprache. Marburg: Lebenshilfe-Verlag 2018.

Literaturhaus (2019): In: https://literaturhaus-frankfurt.de/programm/termine/literatur-in-einfacher-sprache2019-06-26/ (16.02.2022).

Lüthen, Alexandra (2019): Allen eine Chance. Warum wir Leichte Sprache brauchen. Berlin: Dudenverlag. Lüthen, Alexandra (2018): »Herzliebe«. In: Lebenshilfe Berlin (Hg.), S. 63-74.

Lypp, Maria (1984): Einfachheit als Kategorie der Kinderliteratur. Frankfurt a.M.: dipa (Jugend und Medien 9).

Maaß, Christina (2015): Leichte Sprache. Das Regelbuch. Berlin: LIT (Barrierefreie Kommunikation 1).

Mesch, Birgit (2020): »Nicht einfach klassisch - dafür klassisch einfach leicht. Klassiker für Kinder und Jugendliche zwischen leichter, einfacher und poetischer Sprachvarietät«. In: Jörn Brüggemann/Birgit Mesch (Hg.): Sprache als Herausforderung - Literatur als Ziel: Sprachsensible Zugänge zur Kinderund Jugendliteratur. 2 Bde. Baltmannsweiler: Schneider Verlag Hohengehren, Bd. 1, S. 33-52.

Miestamo, Matti (2008): »Grammatical complexity in a cross-linguistic perspective«. In: Matti Miestamo/ Kaius Sinnemäki/Fred Karlsson (Hg.): Language Complexity. Typology, Contact, Change. Amsterdam/Philadelphia: Benjamins (Studies in Language Companion Series 94), S. 23-41.

NDR (2020): Märchen in Leichter Sprache. In: https://www.ndr.de/fernsehen/service/leichte_sprache/ Maerchen-in-Leichter-Sprache, maerchenleichtesprache100.html (16.02.2022).

O’Sullivan, Emer (2016): »Einfachheit im (kinder)literaturtheoretischen Diskurs«. In: Eva Burwitz-Melzer/Emer O'Sullivan (Hg): Einfachheit in der Kinder- und Jugendliteratur. Ein Gewinn für den Fremdsprachenunterricht. Wien: Prasens, S. 17-32.

Piper (2020): In: https://www.piper.de/buecher/lies-das-buch-isbn-978-3-492-07032-4 (16.02.2022).

Pohl, Thorsten (2017): »Komplexität als Operationalisierungsdimension konzeptioneller Schriftlichkeit in Untersuchungen zum Unterrichtsdiskurs«. In: Hennig (Hg.), S. 253-280.

Pressemitteilung (2016): Frankfurt, deine Geschichte. Literatur in Einfacher Sprache. Pressemitteilung 9.9.2016. In: https://www.frankfurt-inklusiv.de/fileadmin/user_upload/download_inklusion/ Materialien/pi_frankfurt_deine_geschichte_literatur_in_einfacher_sprache_9_9_16.pdf (16.02.2022).

Rosebrock, Cornelia (2015): »Der Mut zur Einfalt - Vereinfachte Klassikerausgaben für den Schulgebrauch«. In: Didaktik Deutsch. Halbjahresschrift für die Didaktik der deutschen Sprache und Literatur 38, S. 33-39.

Rosebrock, Cornelia (2019): »Leichte Texte«. In: Christiane Hochstadt/Ralph Olsen (Hg.): Handbuch Deutschunterricht und Inklusion. Weinheim: Beltz, S. 93-110.

Sampson, Geoffrey/Gil, David/Trudgill, Peter (Hg.) (2009): Language Complexity as an Evolving Variable. Oxford: University Press (Studies in the Evolution of Language 13).

Schoch, Julia (2020): »Ich verlasse dich«. In: Hückstädt (Hg.), S. 139-154.

Sklovskij, Viktor (1969): »Die Kunst als Verfahren« [1916]. In: Jurij Striedter (Hg.): Russischer Formalismus. Texte zur allgemeinen Literaturtheorie und zur Theorie der Prosa. München: Wilhelm Fink, S. 5-35.

Spiegel, Carmen/Spranz-Fogasy, Thomas (2001): »Aufbau und Abfolge von Gesprächsphasen«. In: Klaus Brinker/Gerd Antos/Wolfgang Heinemann/Sven F. Sager (Hg.): Text- und Gesprächslinguistik. Ein internationales Handbuch zeitgenössischer Forschung./Linguistics of text and conversation. Berlin/New York: de Gruyter (Handbücher zur Sprach- und Kommunikationswissenschaft 16/2), S. 1241-1252.

Topalovic, Elvira/Diederichs, Lara (2020): »Sprachliches und literarisches Lernen mit Texten in einfacher Sprache: Deutschdidaktische Kontroversen am Beispiel des Romans Tschick«. In: Jörn Brüggemann/Birgit Mesch (Hg.): Sprache als Herausforderung - Literatur als Ziel: Sprachsensible Zugänge zur Kinder- und Jugendliteratur. 2 Bde. Baltmannsweiler: Schneider Verlag Hohengehren, Bd. 1, S. 97-113. 
Übereinkommen (2017): Übereinkommen über die Rechte von Menschen mit Behinderungen. Convention of the United Nations on the rights of persons with disabilities, amtliche, gemeinsame Übersetzung von Deutschland, Österreich, Schweiz und Lichtenstein. Hg. von der Beauftragten der Bundesregierung für die Belange von Menschen mit Behinderungen. Berlin.

Weimar, Klaus (2000): »Literatur«. In: Harald Fricke (Hg.): Reallexikon der deutschen Literaturwissenschaft. Bd. 2. Berlin/New York: de Gruyter, S. 443-448.

Wisotzki, Nadine (2021): Die Kunst der Einfachheit. Standortbestimmungen in der deutschen Gegenwartsliteratur. Judith Hermann - Peter Stamm - Robert Seethaler. Bielefeld: transcript (Gegenwartsliteratur 10).

Zeman, Sonja (2017): »Wie fasst man ein Phantom? Zur Komplexität semantischer Komplexität«. In: Hennig (Hg.), S. 53-72.

Zymner, Rüdiger ( $\left.{ }^{3} 2007\right)$ : »Metapher «. In: Dieter Burdorf/Christoph Fasbender/Burkhard Moennighoff (Hg.): Metzler Lexikon Literatur. Begriffe und Definitionen. Stuttgart/Weimar: Metzler, S. 494-495. 